\title{
Sensitivity equation method for the Navier-Stokes equations applied to uncertainty propagation
}

\author{
Camilla Fiorini $^{1}$, Bruno Després ${ }^{1}$, and Maria Adela Puscas ${ }^{2}$ \\ ${ }^{1}$ LJLL, Sorbonne Université, Paris, France. \\ ${ }^{2}$ CEA, Centre de Saclay, DEN/SAC/DANS/DM2S/STMF/LMSF, Saclay,
} France.

\begin{abstract}
This works deals with sensitivity analysis for the Navier-Stokes equations. The aim is to provide an estimate of the variance of the velocity field when some of the parameters are uncertain and then to use the variance to compute confidence intervals for the output of the model. First, we introduce the physical model and analyse its stability. The sensitivity equations are derived, and their stability analysed as well. We propose a finite elementvolume numerical scheme for the state and the sensitivity, which is integrated into the open-source industrial code TrioCFD. Finally, we present some numerical results: a steady and an unsteady test case for the channel flow problem are investigated. For the steady case, we compare the results to the Monte Carlo method and show how the sensitivity analysis technique succeeds in providing very accurate estimates of the variance. For the unsteady case, a new filtering procedure is proposed to deal with a sensitivity that grows in time. The filtered sensitivity is then used to compute the variance of the output and to provide confidence intervals.
\end{abstract}

\section{Introduction}

Sensitivity analysis (SA) studies how changes in the input of a model affect the output, and it is essential for many engineering applications, such as uncertainty quantification, optimal design, and to answer what if questions, i.e. what happens to the solution of the model if the input parameters change. These tasks can be performed in many different ways, depending on the nature of the model considered. In this work, we consider systems that can be modelled with partial differential equations (PDEs). The sensitivity variable itself is defined as the derivative of the state (i.e., the output of the model) with respect to the parameters of interest.

In the framework of PDEs, one can distinguish two main classes of methods: the differentiate-then-discretise methods and the discretise-then-differentiate methods. As the names say, in the first case the state model is formally differentiated with respect to the parameter of interest, providing an analytical sensitivity system which can then be discretised with the most appropriate numerical scheme. The second class of methods swaps the two steps which, in the general case, are not commutative. A detailed comparison between the two classes of methods can be found in [Gun03] for optimisation problems. In this work, we focus on the first class, and, in particular, on the continuous sensitivity equation method [BB97, DPB06, DP06, CDF18, FCD19].

The main aim of this work is to give an estimate of the variance of the solution of the Navier-Stokes equations when there are uncertain parameters and then to use the estimated variance to compute confidence intervals. This goes under the name of forward uncertainty propagation, which is part of the broader field of uncertainty quantification (UQ). Many strategies and techniques have been proposed in the literature to tackle UQ 
problems, particularly in the case of PDEs models: Monte Carlo method [RC13], polynomial chaos [Wal03, XK03, KM06, DPL13], random space partition [AC13], to name but a few. A review of these methods applied to fluid dynamics problems can be found in [WH02].

Methods of uncertainty propagation based on SA are particularly efficient in terms of computational time if compared, for instance, to methods like Monte Carlo. However, since $\mathrm{SA}$ is based on Taylor expansions of the state variable with respect to the parameter of interest, these methods are intrinsically local [Del14] : they can be used only for random variables with a small variance. The Monte Carlo approach does not require this assumption; however, it is not applicable for realistic unsteady test cases in $2 D$ and $3 D$, due to its high computational cost. In this work we propose an approach based on the sensitivity equation method: under the hypothesis of small variance of the input parameters, we can provide a first-order estimate of the variance of the solution at a reasonable computational cost.

Two declinations of the channel flow test case (i.e. study of the flow past an obstacle) are investigated: a steady $(R e=25)$ and an unsteady $(R e=100)$ test case. For the steady test case, a detailed comparison with the Monte Carlo method is performed: the variance of the output is first estimated using a classical Monte Carlo technique and then with the sensitivity analysis method. The results are extremely accurate. The unsteady test case presents a sensitivity which grows in time: this is caused by the fact that the parameter considered has an infuence on the frequency of vortex shedding. This problem is also described in [HEPB04] for a similar test case to the one considered here: to deal with it, they chose a pulsating inflow velocity, which imposes the frequency of vortex shedding. However, this choice is not suitable for all applications. Therefore we propose a new filtering technique, which allows us to recover the periodic physical part of the sensitivity.

The main original contributions of this work are: (a), to establish the stability in the norm $L^{\infty}\left(0, T ; L^{2}(\Omega)\right) \cap L^{2}\left(0, T ; H^{1}(\Omega)\right)$ for the state and for the sensitivity, we provide an explicit function adapted to the specific geometry to homogenise the Dirichlet boundary conditions (b), we detail the discretisation of the method within a finite element-volume (FEV) framework, well adapted to the open-source industrial code TrioCFD; (c), to be able to remove the phase dependency in the sensitivity signals in the case of pulsating flows, typically in the Von Karman vortex street, we propose and justify a new filtering technique. These results are also validated with a detailed comparison with Monte Carlo simulations.

The paper is organised as follows: in section 2 we present the physical model for the state and derive the sensitivity equations. Stability estimates are provided for both the state and the sensitivity for the prescribed boundary conditions. To do that, we introduce a function to homogenise the Dirichlet boundary conditions: the computation of such a function is explicit and detailed in appendix A. In section 3, we design a FEV method for the sensitivity, which is adapted to the open-source industrial code TrioCFD [DC]. In section 4 , the code implemented for the sensitivity is rigorously validated. In section 5 , we show how the sensitivity can be used to give a first order estimate of the variance of the model output; then the estimated variance is used to compute confidence intervals for the output of the model. In section 6 , the numerical results are presented and discussed.

\section{The physical model}

In this section, we present the physical model as well as some stability estimates for it and its sensitivity.

\subsection{The state equations}

Let us consider the domain $\Omega$ in Figure 1: it is a channel with walls on the top and the bottom and an obstacle of square section at distance $x_{D}$ from the inflow boundary. The Navier-Stokes system and the boundary conditions for this domain are: 


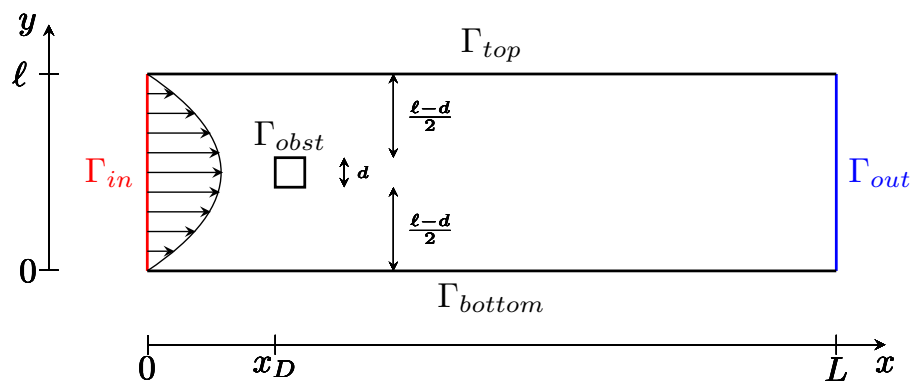

Figure 1: Domain for the first test case.

$$
\begin{cases}\partial_{t} \mathbf{u}-\nu \Delta \mathbf{u}+(\mathbf{u} \cdot \nabla) \mathbf{u}+\nabla p=\mathbf{f} & \Omega, t>0 \\ \nabla \cdot \mathbf{u}=0 & \Omega, t>0, \\ \mathbf{u}(\mathbf{x}, 0)=0 & \Omega, t=0, \\ \mathbf{u}=-g(y) \mathbf{n} & \text { on } \Gamma_{\text {in }}, \\ \mathbf{u}=0 & \text { on } \Gamma_{w}=\Gamma_{\text {obst }} \cup \Gamma_{\text {top }} \cup \Gamma_{\text {bottom }}, \\ (\nu \nabla \mathbf{u}-p I) \mathbf{n}=0 & \text { on } \Gamma_{\text {out }},\end{cases}
$$

where $\mathbf{u}=\left(u^{x}, u^{y}\right)^{t}$ is the velocity, $p$ is the pressure, $\mathbf{f}$ the external force and $g(y)$ the prescribed inflow condition. The first equation models the conservation of the momentum and the second one the conservation of the mass. In the following, they will be referred to as, respectively, the momentum equation and the mass equation. We impose no slip boundary condition of the walls of the domain, a prescribed velocity at the inflow and a homogeneous Neumann boundary condition at the outflow.

Remark: the outflow of the domain is not a physical boundary: the physical domain considered is, in some sense, infinite. The outflow boundary is imposed only for numerical computations: this means that we can always choose it far enough from the obstacle in order to have no recirculation at the outflow and therefore $\left.\mathbf{u} \cdot \mathbf{n}\right|_{\Gamma_{\text {out }}} \geqslant 0$. In [BZD00] one can find a detailed study on the dependence of the recirculation length on Reynolds number for a confined flow past a square obstacle. We use this condition as hypothesis to prove the stability of the state system.

In the following proposition, we provide a stability estimate for the solution $\mathbf{u}$. To do that, we introduce a function $\mathbf{R}_{g}$, which we use to homogenise the inflow boundary conditions. This is also known as a lifting procedure in the mathematical literature.

Proposition 1. Let $\mathbf{R}_{g}$ be a sufficiently smooth ${ }^{1}$ stationary $^{2}$ function such that $\nabla \cdot \mathbf{R}_{g}=$ 0 in $\Omega, \mathbf{R}_{g}=\mathbf{u}$ on $\Gamma_{\text {in }} \cup \Gamma_{w}$, and $\left.\nabla \mathbf{R}_{g} \mathbf{n}\right|_{\Gamma_{\text {out }}}=0$. Then, if $\mathbf{u} \cdot \mathbf{n} \mid \geqslant 0$ on $\Gamma_{\text {out }}$ and $\tilde{\mathbf{f}}$ is stationary the following stability estimate holds:

$$
\|\mathbf{u}\|^{2} \leqslant\left\|\mathbf{R}_{g}\right\|^{2}+\|\tilde{\mathbf{f}}(t)\|^{2} t+K\left(\mathbf{R}_{g}, \tilde{\mathbf{f}}\right) e^{2 t\left\|\nabla \mathbf{R}_{g}\right\|_{L^{\infty}}},
$$

where $\tilde{\mathbf{f}}=\mathbf{f}+\nu \Delta \mathbf{R}_{g}-\left(\mathbf{R}_{g} \cdot \nabla\right) \mathbf{R}_{g}$, and the norm $\|\cdot\|$ is defined as follows:

$$
\|\mathbf{u}\|^{2}:=\|\mathbf{u}(T)\|^{2}+2 \nu \int_{0}^{T}\|\nabla \mathbf{u}(t)\|^{2} d t .
$$

Proof. In order to deal with the boundary terms, we start by homogenising the boundary conditions $\tilde{\mathbf{u}}=\mathbf{u}-\mathbf{R}_{g}$. We have: $\|\mathbf{u}\| \leqslant\|\tilde{\mathbf{u}}\|+\left\|\mathbf{R}_{g}\right\| \|$, therefore, since $\mathbf{R}_{g}$ is regular, controlling $\tilde{\mathbf{u}}$ is equivalent to controlling $\mathbf{u}$.

\footnotetext{
${ }^{1}$ See section A for how to compute such a function.

${ }^{2}$ If the boundary conditions depend on time, $\mathbf{R}_{g}$ cannot be taken stationary.
} 
The equations for $\tilde{\mathbf{u}}$ are:

$$
\begin{cases}\partial_{t} \tilde{\mathbf{u}}-\nu \Delta \tilde{\mathbf{u}}+\left(\left(\tilde{\mathbf{u}}+\mathbf{R}_{g}\right) \cdot \nabla\right) \tilde{\mathbf{u}}+(\tilde{\mathbf{u}} \cdot \nabla) \mathbf{R}_{g}+\nabla p=\tilde{\mathbf{f}} & \Omega, t>0, \\ \nabla \cdot \tilde{\mathbf{u}}=0 & \Omega, t>0, \\ \tilde{\mathbf{u}}(\mathbf{x}, 0)=-\mathbf{R}_{g} & \Omega, t=0, \\ \tilde{\mathbf{u}}=0 & \text { on } \Gamma_{\text {in }} \cup \Gamma_{w}, \\ (\nu \nabla \tilde{\mathbf{u}}-p I) \mathbf{n}=-\nu \nabla \mathbf{R}_{g} \mathbf{n}=0 & \text { on } \Gamma_{\text {out }} .\end{cases}
$$

To obtain the stability estimate, we multiply by $\tilde{\mathbf{u}}$ and integrate by parts:

$$
\begin{array}{r}
\int_{\Omega} \partial_{t} \tilde{\mathbf{u}} \cdot \tilde{\mathbf{u}}+\nu \int_{\Omega} \nabla \tilde{\mathbf{u}}: \nabla \tilde{\mathbf{u}}+\int_{\Omega}\left[\left(\left(\tilde{\mathbf{u}}+\mathbf{R}_{g}\right) \cdot \nabla\right) \tilde{\mathbf{u}}\right] \cdot \tilde{\mathbf{u}}+\int_{\Omega}\left[(\tilde{\mathbf{u}} \cdot \nabla) \mathbf{R}_{g}\right] \cdot \tilde{\mathbf{u}} \\
-\int_{\Gamma_{i n}}(\nu \nabla \tilde{\mathbf{u}}-p I) \mathbf{n} \cdot \tilde{\mathbf{u}}=\int_{\Omega} \tilde{\mathbf{f}} \cdot \tilde{\mathbf{u}} .
\end{array}
$$

The nonlinear term can be rewritten and integrated by parts as follows:

$$
\int_{\Omega}\left[\left(\left(\tilde{\mathbf{u}}+\mathbf{R}_{g}\right) \cdot \nabla\right) \tilde{\mathbf{u}}\right] \cdot \tilde{\mathbf{u}}=\int_{\Omega}\left(\tilde{\mathbf{u}}+\mathbf{R}_{g}\right) \cdot \nabla\left(\frac{|\tilde{\mathbf{u}}|^{2}}{2}\right)=\int_{\partial \Omega}\left(\tilde{\mathbf{u}}+\mathbf{R}_{g}\right) \cdot \mathbf{n} \frac{|\tilde{\mathbf{u}}|^{2}}{2},
$$

where we used the second equation $\nabla \cdot\left(\tilde{\mathbf{u}}+\mathbf{R}_{g}\right)=\nabla \cdot \mathbf{u}=0$. Therefore, using the boundary conditions, one has:

$$
\frac{1}{2} \frac{d}{d t}\|\tilde{\mathbf{u}}(t)\|^{2}+\nu\|\nabla \tilde{\mathbf{u}}\|^{2}+\int_{\Gamma_{\text {out }}}\left(\tilde{\mathbf{u}}+\mathbf{R}_{g}\right) \cdot \mathbf{n} \frac{\tilde{\mathbf{u}}^{2}}{2}+\int_{\Omega}\left[(\tilde{\mathbf{u}} \cdot \nabla) \mathbf{R}_{g}\right] \cdot \tilde{\mathbf{u}}=\int_{\Omega} \tilde{\mathbf{f}} \cdot \tilde{\mathbf{u}},
$$

where $\|\cdot\|$ is the $L^{2}$-norm in space.

We use the hypothesis $\left.\left(\tilde{\mathbf{u}}+\mathbf{R}_{g}\right) \cdot \mathbf{n}\right|_{\Gamma_{\text {out }}}=\left.\mathbf{u} \cdot \mathbf{n}\right|_{\Gamma_{\text {out }}} \geqslant 0$ to remove the integral on $\Gamma_{\text {out }}$. Then, since $\mathbf{R}_{g}$ is regular, we have:

$$
-\int_{\Omega}\left[(\tilde{\mathbf{u}} \cdot \nabla) \mathbf{R}_{g}\right] \cdot \tilde{\mathbf{u}} \leqslant\left\|\nabla \mathbf{R}_{g}\right\|_{L^{\infty}}\|\tilde{\mathbf{u}}\|^{2} .
$$

Therefore, we obtain:

$$
\frac{1}{2} \frac{d}{d t}\|\tilde{\mathbf{u}}\|^{2}+\nu\|\nabla \tilde{\mathbf{u}}\|^{2} \leqslant\left\|\nabla \mathbf{R}_{g}\right\|_{L^{\infty}}\|\tilde{\mathbf{u}}\|^{2}+\|\tilde{\mathbf{f}}\|\|\tilde{\mathbf{u}}\| .
$$

The rest of the proof consists of two steps: first, we prove an estimate for $\|\tilde{\mathbf{u}}\|$; then we substitute this results into (3) to obtain the estimate (2). To obtain an estimate for $\|\tilde{\mathbf{u}}\|$, we start from (3), we remove the positive term $\nu\|\nabla \tilde{\mathbf{u}}\|^{2}$, develop the time derivative, and simplify $\|\tilde{\mathbf{u}}\|$, which is positive, obtaining:

$$
\frac{d}{d t}\|\tilde{\mathbf{u}}\| \leqslant\left\|\nabla \mathbf{R}_{g}\right\|_{L^{\infty}}\|\tilde{\mathbf{u}}\|+\|\tilde{\mathbf{f}}\|
$$

which can be rewritten as:

$$
e^{-t\left\|\nabla \mathbf{R}_{g}\right\|_{L^{\infty}}}\left(\frac{d}{d t}\|\tilde{\mathbf{u}}\|-\left\|\nabla \mathbf{R}_{g}\right\|_{L^{\infty}}\|\tilde{\mathbf{u}}\|\right) \leqslant e^{-t\left\|\nabla \mathbf{R}_{g}\right\|_{L^{\infty}}\|\tilde{\mathbf{f}}\| .}
$$

We remark that the left-hand side is the time derivative of $e^{-\left\|\nabla \mathbf{R}_{g}\right\|_{L^{\infty}} t}\|\tilde{\mathbf{u}}\|$. We can integrate and, if $\mathbf{f}$ is constant in time, we obtain:

$$
\begin{array}{r}
\|\tilde{\mathbf{u}}\| \leqslant\left(\|\tilde{\mathbf{u}}(\mathbf{x}, 0)\|+\frac{\|\tilde{\mathbf{f}}\|}{\left\|\nabla \mathbf{R}_{g}\right\|_{L^{\infty}}}\right) e^{t\left\|\nabla \mathbf{R}_{g}\right\|_{L^{\infty}}} \\
=\left(\left\|\mathbf{R}_{g}\right\|+\frac{\|\tilde{\mathbf{f}}\|}{\left\|\nabla \mathbf{R}_{g}\right\|_{L^{\infty}}}\right) e^{t\left\|\nabla \mathbf{R}_{g}\right\|_{L^{\infty}}},
\end{array}
$$

which concludes the first step. For the second step, we start from (3) and use Young's inequality, obtaining:

$$
\frac{1}{2} \frac{d}{d t}\|\tilde{\mathbf{u}}\|^{2}+\nu\|\nabla \tilde{\mathbf{u}}\|^{2} \leqslant\left(\frac{1}{2}+\left\|\nabla \mathbf{R}_{g}\right\|_{L^{\infty}}\right)\|\tilde{\mathbf{u}}\|^{2}+\frac{1}{2}\|\tilde{\mathbf{f}}\|^{2} .
$$


We multiply by 2 and use the estimate (4), obtaining:

$$
\frac{d}{d t}\|\tilde{\mathbf{u}}\|^{2}+2 \nu\|\nabla \tilde{\mathbf{u}}\|^{2} \leqslant\|\tilde{\mathbf{f}}\|^{2}+\left(1+2\left\|\nabla \mathbf{R}_{g}\right\|_{L^{\infty}}\right)\left(\left\|\mathbf{R}_{g}\right\|+\frac{\|\tilde{\mathbf{f}}\|}{\left\|\nabla \mathbf{R}_{g}\right\|_{L^{\infty}}}\right)^{2} e^{2 t\left\|\nabla \mathbf{R}_{g}\right\|_{L^{\infty}}} .
$$

Finally, we can integrate in time, obtaining:

$$
\begin{aligned}
& \|\tilde{\mathbf{u}}\|^{2}+2 \nu \int_{0}^{t}\|\nabla \tilde{\mathbf{u}}(s)\|^{2} d s \\
& \leqslant\left\|\mathbf{R}_{g}\right\|^{2}+\|\tilde{\mathbf{f}}\|^{2} t+\left(1+2\left\|\nabla \mathbf{R}_{g}\right\|_{L^{\infty}}\right)\left(\left\|\mathbf{R}_{g}\right\|+\frac{\|\tilde{\mathbf{f}}\|}{\left\|\nabla \mathbf{R}_{g}\right\|_{L^{\infty}}}\right)^{2} \frac{1}{2\left\|\nabla \mathbf{R}_{g}\right\|_{L^{\infty}}} e^{2 t\left\|\nabla \mathbf{R}_{g}\right\|_{L^{\infty}}} .
\end{aligned}
$$

\subsection{The sensitivity equations}

We now consider $\mathbf{u}$ as a function of space, time and a scalar uncertain parameter $a, \mathbf{u}=$ $\mathbf{u}(\mathbf{x}, t ; a)$ and we write a formal Taylor expansion with respect to $a$ :

$$
\mathbf{u}(\mathbf{x}, t ; a+\delta a)=\sum_{k=0}^{\infty} \mathbf{u}_{k}(\mathbf{x}, t ; a) \delta a^{k}
$$

where $\mathbf{u}_{0}=\mathbf{u}$ and the coefficient $\mathbf{u}_{k}$ is the $k$-th derivative of $\mathbf{u}$ with respect to $a$ :

$$
\mathbf{u}_{k}(\mathbf{x}, t ; a):=\frac{d^{k}}{d a^{k}} \mathbf{u}(\mathbf{x}, t ; a)
$$

and it is called the $k$-th order sensitivity. To consider more than one parameter of interest, the sensitivity should be defined as the gradient of the state with respect to the vector of parameters and a multi-dimensional Taylor expansion would be necessary, but this is not treated in this work. A similar expansion can be done for the pressure $p$, and the data $\mathbf{f}$, d, and $g$. In order to write the equations for the sensitivities, one can replace (5) into (1) and then factorise according to the powers of $\delta a$. For $k=0$ we obtain the state system (1). For $k=1$, we obtain the first order sensitivity equations. In this work, we consider only first-order sensitivity and the notation $\mathbf{u}_{1}=\mathbf{u}_{a}$ will be employed. This choice is common [BB97, DPB06, DP06, CDF18, FCD19] because in most cases first order sensitivities provide enough information. It is possible to consider higher order sensitivities if necessary, but this is not investigated in this work. The first order sensitivity equations, referred to as the sensitivity equations in short, are:

$$
\begin{cases}\partial_{t} \mathbf{u}_{a}-\nu \Delta \mathbf{u}_{a}+\left(\mathbf{u}_{a} \cdot \nabla\right) \mathbf{u}+(\mathbf{u} \cdot \nabla) \mathbf{u}_{a}+\nabla p_{a}=\mathbf{f}_{a} & \Omega, t>0 \\ \nabla \cdot \mathbf{u}_{a}=0 & \Omega, t>0 \\ \mathbf{u}_{a}(\mathbf{x}, 0)=0 & \Omega, t=0 \\ \mathbf{u}_{a}=-g_{a}(y) \mathbf{n} & \text { on } \Gamma_{i n} \\ \mathbf{u}_{a}=0 & \text { on } \Gamma_{w} \\ \left(\nu \nabla \mathbf{u}_{a}-p_{a} I\right) \mathbf{n}=0 & \text { on } \Gamma_{\text {out }}\end{cases}
$$

where $\Gamma_{w}:=\Gamma_{\text {obst }} \cup \Gamma_{\text {top }} \cup \Gamma_{\text {bottom }}$. These are known as the Oseen equations: an introduction on the subject can be found in [Vol14], both for the theoretical and the numerical aspects. A similar problem is investigated, although only from a numerical point of view, in [DP05, DPB06], where they use the sensitivity for shape optimization problems: in their case an expansion of the normal $\mathbf{n}=\sum \mathbf{n}_{k}(\mathbf{x} ; a) \delta a^{k}$ is necessary, which leads to more complicated boundary conditions. Remark: if $\nu$ is considered as the parameter of interest, the second member of the first equation should be $\overline{\mathbf{f}}_{a}:=\mathbf{f}_{a}+\nu_{a} \Delta \mathbf{u}$ and the Neumann boundary condition should have the additional term $\nu_{a} \nabla \mathbf{u} \mathbf{n}$, but this case is not considered in this work. 
We want to obtain a stability estimate for the sensitivity, similar to the one for the state (2). To do this, we need the following hypothesis:

$$
\exists \kappa=\kappa(\mathbf{u}, \Omega)>0:\left|\int_{\Omega}\left[\left(\mathbf{u}_{a} \cdot \nabla\right) \mathbf{u}\right] \cdot \mathbf{u}_{a}\right| \leqslant \kappa\left\|\mathbf{u}_{a}\right\|^{2} .
$$

We remark that hypothesis (7) is trivial if the $L^{\infty}$-norm of the gradient $\nabla \mathbf{u}$ is controlled (we would have $\kappa=\|\nabla \mathbf{u}\|_{L^{\infty}}$ ). However, in the time dependent case we have a control only on $\int_{0}^{t}\|\nabla \mathbf{u}(s)\|^{2} d s$, which is not sufficient. A similar hypothesis (although less restrictive than ours) can be found in [Ray07]: in the section about linearised Navier-Stokes equations around an instationary state, they suppose

$$
\mathbf{u} \in L^{2}\left(0, T ; H^{1}(\Omega)\right) \cap L^{\infty}\left(0, T ; L^{4}(\Omega)\right) .
$$

From the estimate (2) and the triangular inequality we only have

$$
\mathbf{u} \in L^{2}\left(0, T ; H^{1}(\Omega)\right) \cap L^{\infty}\left(0, T ; L^{2}(\Omega)\right) .
$$

Asking for $\mathbf{u} \in L^{4}(\Omega) \forall t$, would imply having a control on $\|\nabla \mathbf{u}\| \forall t$, because $H^{1}(\Omega) \subset L^{4}(\Omega)$ in 2 dimensions (cf. Corollary 9.11 from [Bre10]).

In the following proposition, we provide a stability estimate for the sensitivity.

Proposition 2. Let $\mathbf{R}_{g_{a}}$ be a sufficiently smooth stationary function such that $\nabla \cdot \mathbf{R}_{g_{a}}=$ 0 in $\Omega, \mathbf{R}_{g_{a}}=\mathbf{u}_{a}$ on $\Gamma_{\text {in }} \cup \Gamma_{w}$, and $\left.\nabla \mathbf{R}_{g_{a}} \mathbf{n}\right|_{\Gamma_{\text {out }}}=0$ Then, if $\mathbf{u} \cdot \mathbf{n} \mid \geqslant 0$ on $\Gamma_{\text {out }}$ and under the hypothesis (7), the following stability estimate holds:

$$
\left\|\mathbf{u}_{a}\right\|^{2} \leqslant\left\|\mathbf{R}_{g_{a}}\right\|^{2}+\left\|\tilde{\mathbf{f}}_{a}\right\|^{2} t+C\left(\mathbf{R}_{g_{a}}, \tilde{\mathbf{f}}_{a}, \kappa\right) e^{2 \kappa t},,
$$

where $\tilde{\mathbf{f}}_{a}=\mathbf{f}_{a}+\nu \Delta \mathbf{R}_{g_{a}}-\left(\mathbf{R}_{g_{a}} \cdot \nabla\right) \mathbf{R}_{g_{a}}$.

Proof. In order to deal with the boundary terms on $\Gamma_{i n}$, we start by homogenising the boundary conditions, as we did earlier for the state equations. We define a new variable $\tilde{\mathbf{u}}_{a}=\mathbf{u}_{a}-\mathbf{R}_{g_{a}}$. The new variable $\tilde{\mathbf{u}}_{a}$ verifies the following equations:

$$
\begin{cases}\partial_{t} \tilde{\mathbf{u}}_{a}-\nu \Delta \tilde{\mathbf{u}}_{a}+\left(\tilde{\mathbf{u}}_{a} \cdot \nabla\right) \mathbf{u}+(\mathbf{u} \cdot \nabla) \tilde{\mathbf{u}}_{a}+\nabla p_{a}=\tilde{\mathbf{f}}_{a} & \Omega, t>0, \\ \nabla \cdot \tilde{\mathbf{u}}_{a}=0 & \Omega, t>0, \\ \tilde{\mathbf{u}}_{a}(\mathbf{x}, 0)=-\mathbf{R}_{g_{a}} & \Omega, t=0, \\ \tilde{\mathbf{u}}_{a}=0 & \text { on } \Gamma_{i n} \cup \Gamma_{w} \\ \left(\nu \nabla \tilde{\mathbf{u}}_{a}-p_{a} I\right) \mathbf{n}=-\nu \nabla \mathbf{R}_{g_{a}} \mathbf{n}=0 & \text { on } \Gamma_{\text {out }} .\end{cases}
$$

We can multiply by $\mathbf{u}_{a}$ and integrate by parts as done for the state, and we obtain:

$$
\frac{1}{2} \frac{d}{d t}\left\|\tilde{\mathbf{u}_{a}}\right\|^{2}+\nu\left\|\nabla \tilde{\mathbf{u}_{a}}\right\|^{2}+\int_{\Omega}\left[\left(\tilde{\mathbf{u}_{a}} \cdot \nabla\right) \mathbf{u}\right] \cdot \tilde{\mathbf{u}_{a}}+\int_{\Omega}\left[(\mathbf{u} \cdot \nabla) \tilde{\mathbf{u}_{a}}\right] \cdot \tilde{\mathbf{u}_{a}}=\int_{\Omega} \mathbf{f}_{a} \mathbf{u}_{a}
$$

All the integrals on the boundary are zero thanks to the boundary conditions. For the first integral, we use hypothesis (7) and obtain

$$
\int_{\Omega}\left[\left(\mathbf{u}_{a} \cdot \nabla\right) \mathbf{u}\right] \cdot \mathbf{u}_{a} \geqslant-\kappa\left\|\mathbf{u}_{a}\right\|^{2} .
$$

The second integral can be treated as we did for the nonlinear term of the state, obtaining:

$$
\int_{\Omega}\left[(\mathbf{u} \cdot \nabla) \tilde{\mathbf{u}_{a}}\right] \cdot \tilde{\mathbf{u}_{a}}=\int_{\Omega} \mathbf{u} \cdot \nabla\left(\frac{\mid \tilde{\left.\mathbf{u}_{a}\right|^{2}}}{2}\right)=\int_{\Gamma_{\text {out }}} \mathbf{u} \cdot \mathbf{n} \frac{\left|\mathbf{u}_{a}\right|^{2}}{2} \geqslant 0 .
$$

By plugging (9) and (10) into (8), we have:

$$
\frac{1}{2} \frac{d}{d t}\left\|\tilde{\mathbf{u}_{a}}\right\|^{2}+\nu\left\|\nabla \tilde{\mathbf{u}_{a}}\right\|^{2} \leqslant \kappa\left\|\tilde{\mathbf{u}}_{a}\right\|^{2}+\left\|\tilde{\mathbf{f}}_{a}\right\|\left\|\tilde{\mathbf{u}_{a}}\right\|,
$$

which has exactly the same structure of (3). Therefore, using the same technique, we obtain:

$$
\left\|\tilde{\mathbf{u}_{a}}\right\|^{2}+2 \nu \int_{0}^{t}\left\|\nabla \tilde{\mathbf{u}_{a}}(s)\right\|^{2} d s \leqslant\left\|\mathbf{R}_{g_{a}}\right\|^{2}+\left\|\tilde{\mathbf{f}}_{a}\right\|^{2} t+\frac{1+2 \kappa}{2 \kappa}\left(\left\|\mathbf{R}_{g_{a}}\right\|+\frac{\left\|\tilde{\mathbf{f}}_{a}\right\|}{\kappa}\right)^{2} e^{2 \kappa t} .
$$




\section{Numerical schemes}

In order to discretise the systems (1) and (6), we use a finite element-volume (FEV) method in space and forward Euler in time. For more details about FEV, cf. [Emo92, Hei03, APFC17, ABF15]. The numerical schemes described below are implemented in the industrial open source code TRUST TrioCFD [DC].

\subsection{Spatial discretisation}

Here, we recall the main ideas of the FEV method for the state and we adapt it to the sensitivity equations. For this purpose, in this subsection we only consider the spatial part of the systems (1) and (6) (i.e. $\partial_{t} \mathbf{u}=\partial_{t} \mathbf{u}_{a}=0$ ). Let $\Gamma_{D}=\Gamma_{i n} \cup \Gamma_{w}$ be the Dirichlet boundary and $\Gamma_{N}=\Gamma_{\text {out }}$ the Neumann boundary. Let $\mathcal{T}_{h}$ be a triangulation of the domain $\Omega$ compatible with the boundary conditions, and $K_{j} \in \mathcal{T}_{h}$ a triangle $\left(j=1, \ldots, N_{T}\right)$ : a triangulation is said to be compatible with the boundary conditions when, if a triangle $K_{j}$ has a side on the boundary, than that side belongs entirely to $\Gamma_{D}$ or entirely to $\Gamma_{N}$. We denote with $\mathbf{x}_{i}$ the nodes $\left(i=1, \ldots, N_{N}\right)$, which are the middle points of the edges of the triangles. A control volume $\omega_{i}$ is associated with each node (cf. Figure 2 for the definition of $\left.\omega_{i}\right)$.

We introduce the following spaces:

$$
\begin{gathered}
Q_{h}=\left\{q_{h}: \forall K \in \mathcal{T}_{h},\left.q_{h}\right|_{K} \in P_{0}(K)\right\}, \\
V_{h}=\left\{w_{h} \text { continuous at } \mathbf{x}_{i}: \forall K \in \mathcal{T}_{h},\left.w_{h}\right|_{K} \in P_{1}(K)\right\}, \\
\mathbf{V}_{h}=\left\{\mathbf{w}_{h}=\left(w^{x}, w^{y}\right)^{t}: w^{x}, w^{y} \in V_{h}\right\}=V_{h}^{2} .
\end{gathered}
$$

The space $Q_{h}$ is spanned by the indicator functions of the triangles, $\chi_{K}$, and $V_{h}$ is spanned by $\varphi_{i}(\mathbf{x})$, with $\varphi_{i} \in V_{h}$ and $\varphi_{i}\left(\mathbf{x}_{j}\right)=\delta_{i j}$. We look for an approximate solution for the systems (1) and (6), respectively $\left(\mathbf{u}_{h}, p_{h}\right) \in \mathbf{V}_{h} \times Q_{h}$ and $\left(\mathbf{u}_{a, h}, p_{a, h}\right) \in \mathbf{V}_{h} \times Q_{h}$. The two components of the discrete velocity field and its sensitivity will be denoted $\mathbf{u}_{h}=\left(u_{h}^{x}, u_{h}^{y}\right)^{t}$ and $\mathbf{u}_{a, h}=\left(u_{a, h}^{x}, u_{a, h}^{y}\right)^{t}$.

In order to have a discrete formulation, we integrate the mass equation and its sensitivity over the triangles $K_{j}$ and the momentum equation and its sensitivity over the control volumes $\omega_{i}$ :

$$
\begin{array}{cc}
\int_{\partial K_{j} \backslash \Gamma_{D}} \mathbf{u}_{h} \cdot \mathbf{n}=\int_{\partial K_{j} \cap \Gamma_{i n}} g & \forall K_{j} \in \mathcal{T}_{h}, \\
-\int_{\partial \omega_{i} \backslash \Gamma_{N}}\left(\nu \nabla \mathbf{u}_{h}-p_{h} I\right) \mathbf{n}+\int_{\partial \omega_{i} \backslash \Gamma_{D}}\left(\mathbf{u}_{h} \otimes \mathbf{u}_{h}\right) \mathbf{n}= & \forall \omega_{i}, \\
=\int_{\omega_{i}} \mathbf{f}-\int_{\partial \omega_{i} \cap \Gamma_{i n}}(g \mathbf{n} \otimes g \mathbf{n}) \mathbf{n} & \forall K_{j} \in \mathcal{T}_{h}, \\
\int_{\partial K_{j} \backslash \Gamma_{D}} \mathbf{u}_{a, h} \cdot \mathbf{n}=\int_{\partial K_{j} \cap \Gamma_{i n}} g_{a} & \\
-\int_{\partial \omega_{i} \backslash \Gamma_{N}}\left(\nu \nabla \mathbf{u}_{a, h}-p_{a, h} I\right) \mathbf{n}+\int_{\partial \omega_{i} \backslash \Gamma_{D}}\left(\mathbf{u}_{a, h} \otimes \mathbf{u}_{h}+\mathbf{u}_{h} \otimes \mathbf{u}_{a, h}\right) \mathbf{n}= & \forall \omega_{i} . \\
=\int_{\omega_{i}} \mathbf{f}_{a}-\int_{\partial \omega_{i} \cap \Gamma_{i n}}\left(g_{a} \mathbf{n} \otimes g \mathbf{n}+g \mathbf{n} \otimes g_{a} \mathbf{n}\right) \mathbf{n} &
\end{array}
$$

Then, one can expand $\left(\mathbf{u}_{h}, p_{h}\right)$ and $\left(\mathbf{u}_{a, h}, p_{a, h}\right)$ in the bases of the correspondent spaces as follows:

$$
\begin{aligned}
\mathbf{u}_{h}(\mathbf{x}) & =\sum_{i=1}^{N_{N}} \mathbf{u}_{h}\left(\mathbf{x}_{i}\right) \varphi_{i}(\mathbf{x}), & p_{h}(\mathbf{x}) & =\sum_{j=1}^{N_{T}} p_{h}\left(K_{j}\right) \chi_{K_{j}} \\
\mathbf{u}_{a, h}(\mathbf{x}) & =\sum_{i=1}^{N_{N}} \mathbf{u}_{a, h}\left(\mathbf{x}_{i}\right) \varphi_{i}(\mathbf{x}), & p_{a, h}(\mathbf{x}) & =\sum_{j=1}^{N_{T}} p_{a, h}\left(K_{j}\right) \chi_{K_{j}}
\end{aligned}
$$

We divide the nodes into two sets: the Dirichlet nodes $\mathcal{D}:=\left\{i: \mathbf{x}_{i} \in \Gamma_{D}\right\}$ and all the other nodes which are referred to as the internal nodes $\mathcal{I}:=\left\{i: \mathbf{x}_{i} \in \Omega \cup \Gamma_{N}\right\}$. By plugging (12) 
into (11) for the linear terms and using the identity $(\mathbf{a} \otimes \mathbf{b}) \mathbf{c}=\mathbf{a}(\mathbf{b} \cdot \mathbf{c})$ for the nonlinear terms, we have:

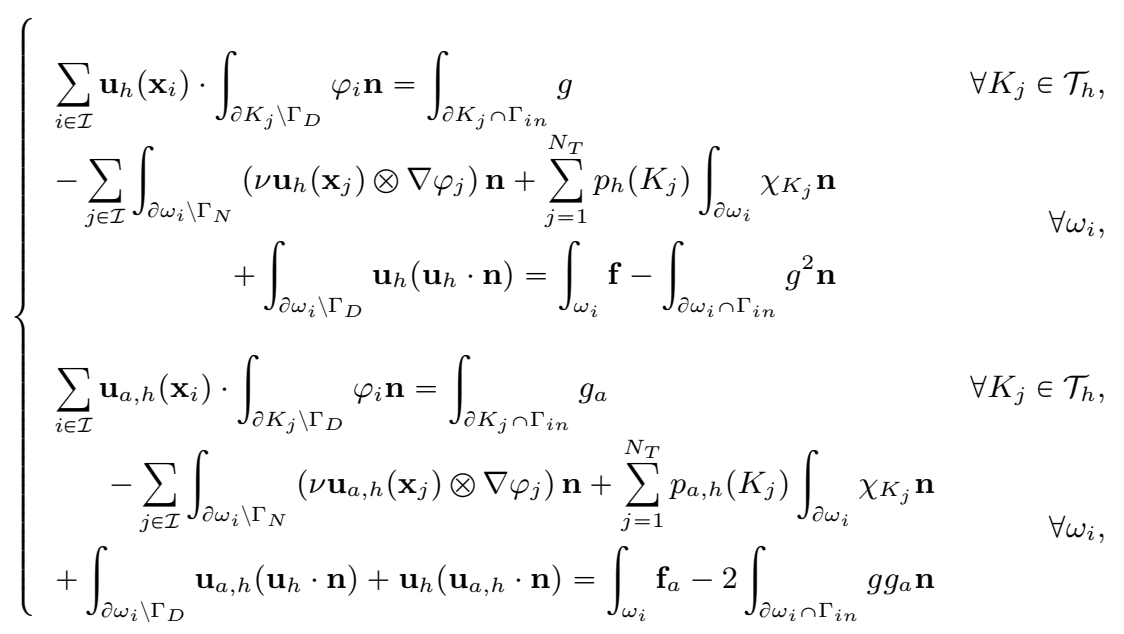

We can define the vectors of unknowns as follows:

$$
\begin{aligned}
& \mathcal{U}_{h}=\left[u_{h}^{x}\left(\mathbf{x}_{i}\right)\right]_{i \in \mathcal{I}}, \quad \mathcal{V}_{h}=\left[u_{h}^{y}\left(\mathbf{x}_{i}\right)\right]_{i \in \mathcal{I}}, \quad \mathbf{P}_{h}=\left[p_{h}\left(K_{j}\right)\right]_{j=1, \ldots, N_{T}}, \quad \mathbf{U}_{h}=\left[\begin{array}{l}
\mathcal{U}_{h} \\
\mathcal{V}_{h}
\end{array}\right], \\
& \mathcal{U}_{a, h}=\left[u_{a, h}^{x}\left(\mathbf{x}_{i}\right)\right]_{i \in \mathcal{I}}, \quad \mathcal{V}_{a, h}=\left[u_{a, h}^{y}\left(\mathbf{x}_{i}\right)\right]_{i \in \mathcal{I}} \quad \mathbf{P}_{a, h}=\left[p_{a, h}\left(K_{j}\right)\right]_{j=1, \ldots, N_{T}}, \quad \mathbf{U}_{a, h}=\left[\begin{array}{l}
\mathcal{U}_{a, h} \\
\mathcal{V}_{a, h}
\end{array}\right] .
\end{aligned}
$$

We introduce the following vectors:

$$
\begin{aligned}
F_{i}^{\ell} & =\int_{\omega_{i}} f^{\ell}-\int_{\partial \omega_{i} \cap \Gamma_{i n}} g^{2} n^{\ell} & D_{i} & =\int_{\partial K_{i} \cap \Gamma_{i n}} g \\
F_{a i}^{\ell} & =\int_{\omega_{i}} f_{a}^{\ell}-2 \int_{\partial \omega_{i} \cap \Gamma_{i n}} g g_{a} n^{\ell} & D_{a i} & =\int_{\partial K_{i} \cap \Gamma_{i n}} g_{a}
\end{aligned}
$$

and the following matrices:

$$
\tilde{A}_{i, j}:=-\int_{\partial \omega_{i} \backslash \Gamma_{N}} \nu \nabla \varphi_{j} \cdot \mathbf{n} \quad B_{i, j}^{\ell}:=\int_{\partial K_{j} \backslash \Gamma_{D}} \varphi_{i} n^{\ell} \quad C_{i, j}^{\ell}:=\int_{\partial \omega_{i} \backslash \Gamma_{N}} \chi_{K_{j}} n^{\ell}
$$

where the superscript $\ell=x, y$ indicates the $x$ - and $y$ - components of the normal vector $\mathbf{n}=\left(n^{x}, n^{y}\right)$, of the state source term $\mathbf{f}=\left(f^{x}, f^{y}\right)$, and of the sensitivity source term $\mathbf{f}_{a}=\left(f_{a}^{x}, f_{a}^{y}\right)$.

For the convection terms, an upwind-type approach is applied: for each edge of $\partial \omega_{i}, \mathbf{u}_{h} \cdot \mathbf{n}$ is computed and, according to its sign, it is multiplied by either $\mathbf{u}_{h}\left(\mathbf{x}_{i}\right)$ or $\mathbf{u}_{h}\left(\mathbf{x}_{k}\right)$, where $\omega_{k}$ is the control volume adjacent to the considered edge. We remark that using an upwind-type scheme leads to a CFL condition [CFL67] on the time step $\Delta t$ when considering the time discretisation: this CFL condition is the same for the state and the sensitivity equations because the transport speed in both equations is $\mathbf{u}$.

For the sake of having a more compact and readable notation, we define $\mathbf{x}_{*}$ as follows:

$$
\mathbf{x}_{*}= \begin{cases}\mathbf{x}_{i} & \text { if } \mathbf{u}_{h} \cdot \mathbf{n}_{i}>0 \text { on } \partial \omega_{i} \cap \partial \omega_{k} \\ \mathbf{x}_{k} & \text { otherwise }\end{cases}
$$

which truly depends on $\mathbf{x}_{i}, \mathbf{x}_{k}$ and $\mathbf{u}_{h}$, but we use a simplified notation. The context will make it non ambiguous. In the same way, we can define $\mathbf{x}_{a *}$ according to the sign of $\mathbf{u}_{a, h} \cdot \mathbf{n}$. Therefore, for the convection term in the state equation, we can write:

$$
\int_{\partial \omega_{i}} \mathbf{u}_{h}\left(\mathbf{u}_{h} \cdot \mathbf{n}\right) \approx \int_{\partial \omega_{i}} \mathbf{u}_{h}\left(\mathbf{x}_{*}\right)\left(\mathbf{u}_{h} \cdot \mathbf{n}\right)=\int_{\partial \omega_{i}} \mathbf{u}_{h}\left(\mathbf{x}_{*}\right)\left(\sum_{j \in \mathcal{I}} \mathbf{u}_{h}\left(\mathbf{x}_{j}\right) \varphi_{j} \cdot \mathbf{n}\right) .
$$


We consider the first component of the vector here above:

$$
\begin{aligned}
\int_{\partial \omega_{i}} u_{h}^{x}\left(\mathbf{x}_{*}\right) & \left(\sum_{j \in \mathcal{I}} \mathbf{u}_{h}\left(\mathbf{x}_{j}\right) \varphi_{j} \cdot \mathbf{n}\right)= \\
& =\sum_{j \in \mathcal{I}} u_{h}^{x}\left(\mathbf{x}_{j}\right) \int_{\partial \omega_{i}} u_{h}^{x}\left(\mathbf{x}_{*}\right) \varphi_{j} n^{x}+\sum_{j \in \mathcal{I}} u_{h}^{y}\left(\mathbf{x}_{j}\right) \int_{\partial \omega_{i}} u_{h}^{x}\left(\mathbf{x}_{*}\right) \varphi_{j} n^{y} .
\end{aligned}
$$

We can define the following matrices:

$$
L_{i, j}^{x}\left(\mathcal{U}_{h}\right)=\int_{\partial \omega_{i}} u_{h}^{x}\left(\mathbf{x}_{*}\right) \varphi_{j} n^{x}, \quad L_{i, j}^{y}\left(\mathcal{U}_{h}\right)=\int_{\partial \omega_{i}} u_{h}^{x}\left(\mathbf{x}_{*}\right) \varphi_{j} n^{y} .
$$

The second component would give as a result $L_{i, j}^{x}\left(\mathcal{V}_{h}\right)$ and $L_{i, j}^{y}\left(\mathcal{V}_{h}\right)$. Concerning the sensitivity, doing the same computations would lead to eight matrices, four of them identical to the ones introduced for the state, and four others as follows:

$$
\begin{array}{ll}
L_{a i, j}^{x}\left(\mathcal{U}_{a, h}\right)=\int_{\partial \omega_{i}} u_{a, h}^{x}\left(\mathbf{x}_{a *}\right) \varphi_{j} n^{x}, & L_{a i, j}^{y}\left(\mathcal{U}_{a, h}\right)=\int_{\partial \omega_{i}} u_{a, h}^{x}\left(\mathbf{x}_{a *}\right) \varphi_{j} n^{y} \\
L_{a i, j}^{x}\left(\mathcal{V}_{a, h}\right)=\int_{\partial \omega_{i}} u_{a, h}^{y}\left(\mathbf{x}_{a *}\right) \varphi_{j} n^{x}, & L_{a i, j}^{y}\left(\mathcal{V}_{a, h}\right)=\int_{\partial \omega_{i}} u_{a, h}^{y}\left(\mathbf{x}_{a *}\right) \varphi_{j} n^{y}
\end{array}
$$

Finally, by introducing the following notation:

$$
\begin{gathered}
A=\left(\begin{array}{cc}
\tilde{A} & 0 \\
0 & \tilde{A}
\end{array}\right), \quad B=\left(\begin{array}{ll}
B^{x} & \left.B^{y}\right),
\end{array} \quad C=\left(\begin{array}{l}
C^{x} \\
C^{y}
\end{array}\right), \quad F=\left(\begin{array}{l}
F^{x} \\
F^{y}
\end{array}\right), \quad F_{a}=\left(\begin{array}{c}
F_{a}^{x} \\
F_{a}^{y}
\end{array}\right),\right. \\
L\left(\mathbf{U}_{h}\right)=\left(\begin{array}{ll}
L^{x}\left(\mathcal{U}_{h}\right) & L^{y}\left(\mathcal{U}_{h}\right) \\
L^{x}\left(\mathcal{V}_{h}\right) & L^{y}\left(\mathcal{V}_{h}\right)
\end{array}\right), \quad L_{a}\left(\mathbf{U}_{a, h}\right)=\left(\begin{array}{ll}
L_{a}^{x}\left(\mathcal{U}_{a, h}\right) & L_{a}^{y}\left(\mathcal{U}_{a, h}\right) \\
L_{a}^{x}\left(\mathcal{V}_{a, h}\right) & L_{a}^{y}\left(\mathcal{V}_{a, h}\right)
\end{array}\right),
\end{gathered}
$$

and observing that $C=B^{t}$, the discrete system can be written in the compact form:

$$
\left\{\begin{array}{l}
A \mathbf{U}_{h}+B^{t} \mathbf{P}_{h}+L\left(\mathbf{U}_{h}\right) \mathbf{U}_{h}=F \\
B \mathbf{U}_{h}=D \\
A \mathbf{U}_{a, h}+B^{t} \mathbf{P}_{a, h}+L_{a}\left(\mathbf{U}_{a, h}\right) \mathbf{U}_{h}+L\left(\mathbf{U}_{h}\right) \mathbf{U}_{a, h}=F_{a} \\
B \mathbf{U}_{a, h}=D_{a}
\end{array}\right.
$$

The first two equations correspond to the state system, and are independent of the last two: the first one is the discretisation of the momentum equation and the second one is the discretisation of the mass equation $\nabla \cdot \mathbf{u}=0$, where the vector $D$ accounts for the Dirichlet boundary conditions. The last two equations correspond to the sensitivity and, in particular, the first of these depends on the solution of the state through the convective matrix $L\left(\mathbf{U}_{h}\right)$.

\subsection{Time discretisation}

For the time scheme, we use a forward Euler discretisation [QSS10] with an implicit treatment of the diffusion operator: an explicit treatment would require a very restrictive time step for stability. Let $t^{n}$ be the $n$-th time step, which is computed according to the CFL condition [CFL67], and let $\mathbf{U}_{h}^{n}$ (respectively $\mathbf{U}_{a, h}^{n}$ ) be an approximation of $\mathbf{u}\left(x, t^{n}\right.$ ) (respectively, $\left.\mathbf{u}_{a}\left(x, t^{n}\right)\right)$. By coupling this with the spatial scheme described in the previous subsection, we obtain the following system:

$$
\left\{\begin{array}{l}
M \frac{\mathbf{U}_{h}^{n+1}-\mathbf{U}_{h}^{n}}{\Delta t}+A \mathbf{U}_{h}^{n+1}+B^{t} \mathbf{P}_{h}^{n+1}+L\left(\mathbf{U}_{h}^{n}\right) \mathbf{U}_{h}^{n}=F^{n}, \\
B \mathbf{U}_{h}^{n+1}=D^{n}, \\
M \frac{\mathbf{U}_{a, h}^{n+1}-\mathbf{U}_{a, h}^{n}}{\Delta t}+A \mathbf{U}_{a, h}^{n+1}+B^{t} \mathbf{P}_{a, h}^{n+1}+L_{a}\left(\mathbf{U}_{a, h}^{n}\right) \mathbf{U}_{h}^{n}+L\left(\mathbf{U}_{h}^{n}\right) \mathbf{U}_{a, h}^{n}=F_{a}^{n}, \\
B \mathbf{U}_{a, h}^{n+1}=D_{a}^{n},
\end{array}\right.
$$




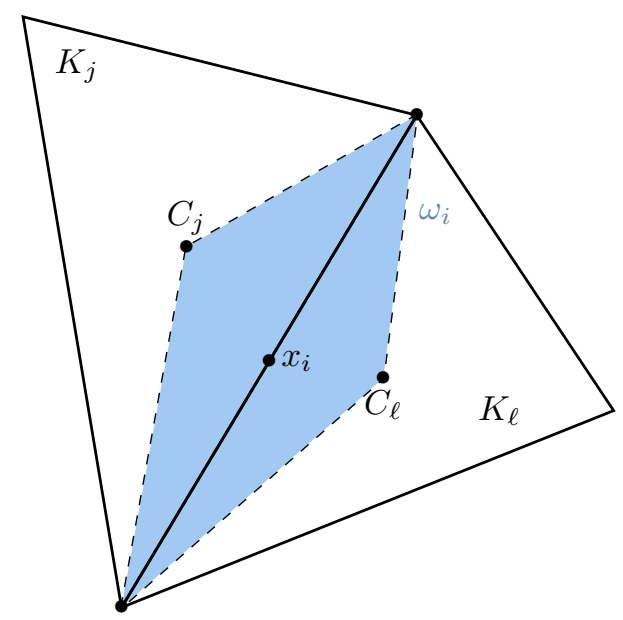

Figure 2: The control volume $\omega_{i}$ associated with the node $\mathbf{x}_{i}$ is shaded in blue. $C_{j}$ and $C_{\ell}$ are the centres of gravity of the two triangles $K_{j}$ and $K_{\ell}$ adjacent to the edge on which $\mathbf{x}_{i}$ lies.

where $M$ is the mass matrix with coefficients $M_{i, j}=\int_{\omega_{i}} \varphi_{j}$. To improve the efficiency in the computations, $M$ is lumped, i.e. it is approximated with a diagonal matrix whose coefficients are $\left|\omega_{i}\right| \delta_{i, j}$. The system (14) is solved with a prediction correction procedure [Cho68, Tem68, APFC17]. First, an intermediate speed $\mathbf{U}_{h}^{*}$ and its sensitivity $\mathbf{U}_{a, h}^{*}$ (which are not solenoidal) are computed by solving the following systems:

$$
\begin{aligned}
\left(I+\Delta t M^{-1} A\right) \mathbf{U}_{h}^{*} & =\mathbf{U}_{h}^{n}-\Delta t M^{-1}\left(L\left(\mathbf{U}_{h}^{n}\right) \mathbf{U}_{h}^{n}+B^{t} \mathbf{P}_{h}^{n}-F^{n}\right), \\
\left(I+\Delta t M^{-1} A\right) \mathbf{U}_{a, h}^{*} & =\mathbf{U}_{a, h}^{n}-\Delta t M^{-1}\left(L_{a}\left(\mathbf{U}_{a, h}^{n}\right) \mathbf{U}_{h}^{n}+L\left(\mathbf{U}_{h}^{n}\right) \mathbf{U}_{a, h}^{n}+B^{t} \mathbf{P}_{a, h}^{n}-F_{a}^{n}\right) .
\end{aligned}
$$

Then, we perform the correction step:

$$
\begin{aligned}
& \mathbf{U}_{h}^{n+1}=\mathbf{U}_{h}^{*}+\Delta t M^{-1} B^{t}\left(\mathbf{P}_{h}^{n+1}-\mathbf{P}_{h}^{n}\right), \\
& \mathbf{U}_{a, h}^{n+1}=\mathbf{U}_{a, h}^{*}+\Delta t M^{-1} B^{t}\left(\mathbf{P}_{a, h}^{n+1}-\mathbf{P}_{a, h}^{n}\right) .
\end{aligned}
$$

Finally, by left multiplying by $B$ and imposing the second and fourth equation of (14), one obtains:

$$
\begin{gathered}
B M^{-1} B^{t} \mathbf{P}_{h}^{n+1}=B M^{-1} B^{t} \mathbf{P}_{h}^{n}+\frac{D^{n+1}-B \mathbf{U}_{h}^{*}}{\Delta t}, \\
B M^{-1} B^{t} \mathbf{P}_{a, h}^{n+1}=B M^{-1} B^{t} \mathbf{P}_{a, h}^{n}+\frac{D_{a}^{n+1}-B \mathbf{U}_{a, h}^{*}}{\Delta t},
\end{gathered}
$$

and $\mathbf{P}_{h}^{n+1}$ and $\mathbf{P}_{a, h}^{n+1}$ can be used to compute $\mathbf{U}_{h}^{n+1}$ and $\mathbf{U}_{a, h}^{n+1}$ from (15). We remark that in order to solve the pressure problem (16), some technical discrete boundary conditions for the pressure are needed. To do this, in the code TrioCFD [DC] the Neumann boundary condition is split into two parts: the pressure and the gradient of the velocity are set to zero independently of each other.

\section{Validation of the implementation of the sensitivity equation}

In order to validate the sensitivity equation method, we consider the following Taylor expansion:

$$
\mathbf{u}(x, T ; a+\delta a)=\mathbf{u}(x, T ; a)+\delta a \mathbf{u}_{a}(x, T ; a)+O\left(\delta a^{2}\right),
$$




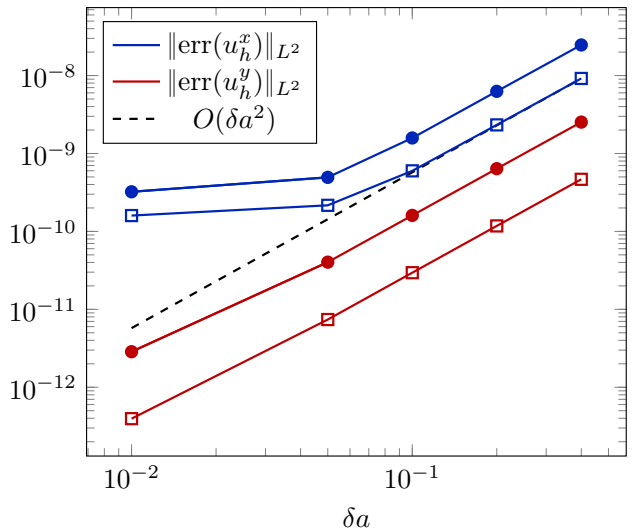

(a) $L^{2}$-norm

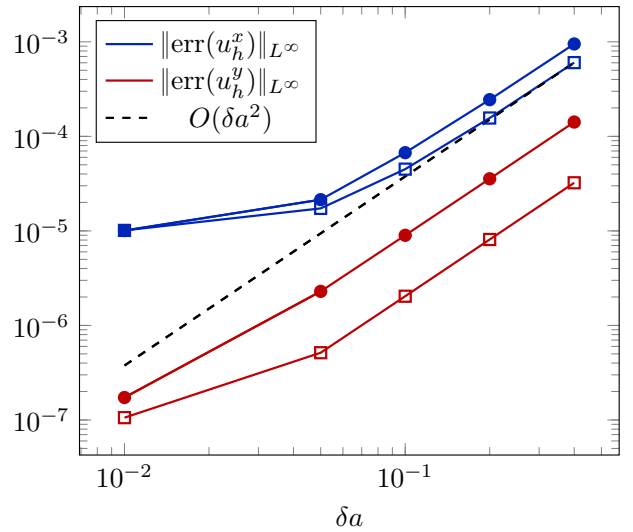

(b) $L^{\infty}$-norm.

Figure 3: Convergence of the SE method. The symbol $\square$ corresponds to the finer mesh $(h=$ $0.001)$, the symbol $\bullet$ to the coarser one $(h=0.002)$.

and we define the following quantity:

$$
\operatorname{err}(\mathbf{u})=\mathbf{u}(x, T ; a+\delta a)-\mathbf{u}(x, T ; a)-\delta a \mathbf{u}_{a}(x, T ; a) .
$$

If the sensitivity equation are correctly solved, one should have $\operatorname{err}(\mathbf{u})=O\left(\delta a^{2}\right)$. We considered a straightforward test case: a channel as the one in Figure 1 but without the obstacle and with a parabolic velocity profile imposed at the inlet:

$$
g(y)=\frac{4 A}{\ell^{2}} y(\ell-y)
$$

The maximal value of the velocity at the inlet is the parameter of interest $a$ (i.e. $a=A$ ). We solved this on two Cartesian grids with $h=0.001$ and $h=0.002$. In Figure 3, we show the $L^{2}$ and $L^{\infty}$ norms for the two components of the velocity on the two different meshes: for larger $\delta a$ we observe the expected convergence rate, but starting at $\delta a \simeq 0.05$ the curve flattens (especially the ones corresponding to the horizontal component of the velocity). This is due to the fact that the quantity shown is $\operatorname{err}\left(\mathbf{u}_{h}\right)$ and not $\operatorname{err}(\mathbf{u})$ : the spatial discretisation is an additional source of error, and this part of the error is constant for a given mesh, therefore the curve tend to a plateau. The plateau value is smaller for finer meshes, as one can observe in Figure 3.

\section{Uncertainty propagation}

In this section, we want to show how the sensitivity can be used to give a first order estimate of the variance of the model output. In this context, the parameter $a$ is a random variable with a known distribution, expected value $\mu_{a}$, and variance $\sigma_{a}^{2}$. Let $X(\mathbf{x}, t ; a)$ be a physical variable (i.e. the horizontal or vertical velocity, or the pressure), whose expected value $\mu_{X}$ and variance $\sigma_{X}^{2}$ we want to estimate. To do this, we start from a Taylor expansion of $X$ with respect to the parameter $a$ centred in the expected value of $a, \mu_{a}$ :

$$
X(\mathbf{x}, t ; a)=X\left(\mathbf{x}, t ; \mu_{a}\right)+\left(a-\mu_{a}\right) X_{a}(\mathbf{x}, t ; a)+o\left(\left|a-\mu_{a}\right|^{2}\right),
$$

where $X_{a}=\partial_{a} X$ is the sensitivity of $X$ with respect to the parameter $a$. Computing the expected value of the right and left-hand side of (17), one obtains the following first order estimate:

$$
\mu_{X}(\mathbf{x}, t)=E[X(\mathbf{x}, t ; a)] \simeq X\left(\mathbf{x}, t ; \mu_{a}\right)+E\left[\left(a-\mu_{a}\right)\right] X_{a}(\mathbf{x}, t ; a)=X\left(\mathbf{x}, t ; \mu_{a}\right) .
$$




\begin{tabular}{c|cccc} 
parameter & $\ell$ & $L$ & $x_{D}$ & $d$ \\
\hline value & 0.7 & 2 & 0.4 & 0.1
\end{tabular}

Table 1: Values of the domain parameters used in simulations.

Using again the Taylor expansion (17) and the estimate just obtained for the average (18), we obtain an estimate of the variance:

$$
\sigma_{X}^{2}(\mathbf{x}, t)=E\left[\left(X(\mathbf{x}, t ; a)-\mu_{X}(\mathbf{x}, t)\right)^{2}\right] \simeq E\left[\left(a-\mu_{a}\right)^{2}\right] X_{a}^{2}(\mathbf{x}, t ; a)=\sigma_{a}^{2} X_{a}^{2}(\mathbf{x}, t ; a) .
$$

The estimates (18)-(19) are valid only where the Taylor expansion (17) holds, i.e. for small variances of the random parameter $\sigma_{a}^{2}$. However, one can have an estimate of the variance with just one simulation of the state and one of the sensitivity, which is a minimal computational cost when compared to methods such as Monte Carlo that require thousands of simulations of the state to estimate the variance. In the general case, when more than one parameter is uncertain, to provide an estimate of the variance, one would need one simulation for the state and as many simulations of the sensitivity as the number of uncertain parameters [Fio18]. This makes the sensitivity approach really affordable and highly competitive when the number of uncertain parameters is small enough. In the next subsection, we compare the results of the SA approach with the well-known Monte Carlo method [RC13].

The estimated variance can be used for multiple purposes. In this work, we use it to compute some confidence intervals for the physical variables, i.e. find an interval $C I_{X}$ such that the probability that $X$ falls into $C I_{X}$ is bigger that $1-\alpha$. Standard choices for $\alpha$ are 0.05 or 0.01 . If the distribution of the random variable $X$ is known, some precise estimates for the extrema of the interval exist. However, SA does not provide any insight of what the distribution of the output is. Therefore we start from Chebyshev inequality [JP12], which states that for any random variable with finite expected value and variance

$$
P\left(\left|X-\mu_{X}\right| \geqslant \lambda\right) \leqslant \frac{\sigma_{X}^{2}}{\lambda^{2}} .
$$

By imposing the desired level for confidence interval, i.e. $\alpha=\frac{\sigma_{X}^{2}}{\lambda^{2}}$, one gets $\lambda=\frac{\sigma_{X}}{\sqrt{\alpha}}$, therefore

$$
C I_{X}=\left[\mu_{X}-\frac{\sigma_{X}}{\sqrt{\alpha}}, \mu_{X}+\frac{\sigma_{X}}{\sqrt{\alpha}}\right] .
$$

\section{$6 \quad$ Numerical results}

The domain used for the numerical simulations is the one in Figure 1, and the values of the parameters are the ones in Table 1 . The equations are solved on the mesh with a spatial step varying from 0.005 to 0.01 . Finally, we consider the following parabolic inflow condition:

$$
g(y)=\frac{4 A}{\ell^{2}} y(y-\ell)
$$

where $A$ is the maximal value of the inflow velocity, and it is the uncertain parameter (i.e. $a=A) . A$ is a Gaussian random variable of average $\mu_{A}$ and variance $\sigma_{A}^{2}$.

\subsection{Steady test case}

For this first test case, we consider a small inflow velocity, $\mu_{A}=0.25$, which corresponds to $R e=25$ and leads to a stationary solution. The probability density function of $A$ is shown in Figure 4: the standard deviation $\sigma_{A}=7.5 \times 10^{-3}$ is small enough to apply the sensitivity method described above to compute the variance of the output.

In Figure 5, we show the numerical results for the horizontal velocity and its sensitivity. For this test case, we were able to make a Monte Carlo approach as well: 1300 simulations 


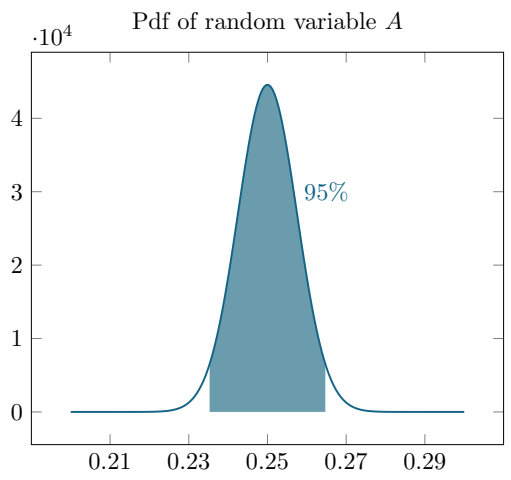

Figure 4: Probability density function of the uncertain parameter $A$.

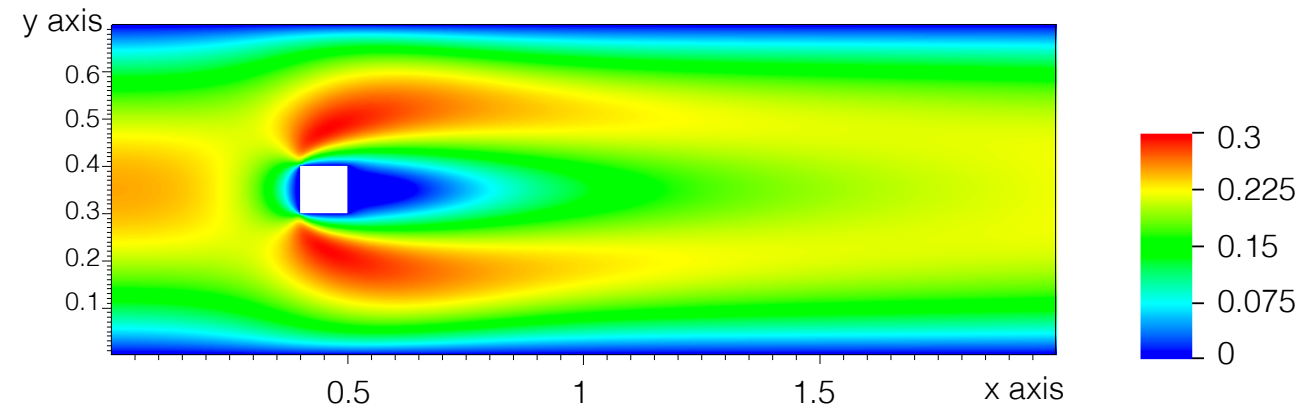

(a) Horizontal velocity

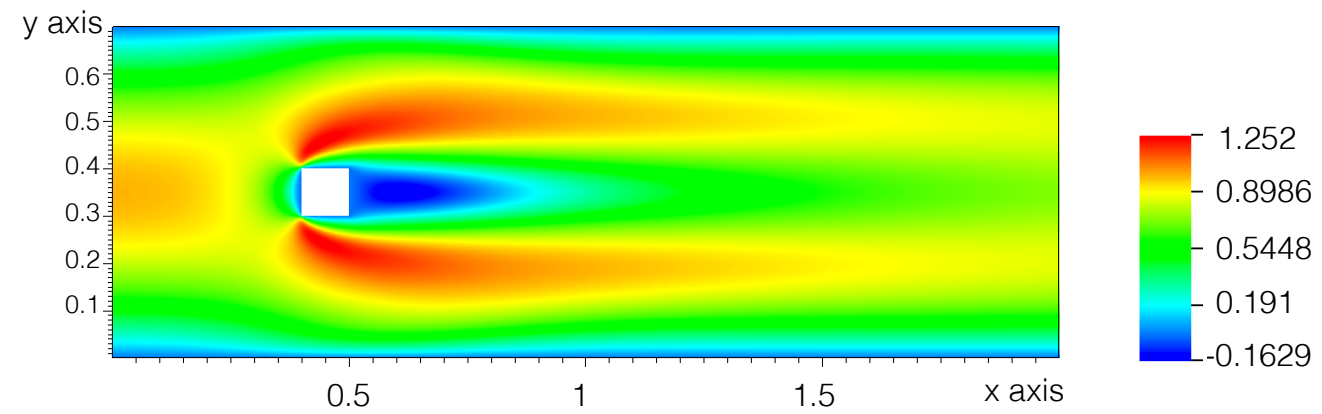

(b) Sensitivity of the horizontal velocity

Figure 5: Results for $R e=25$. 


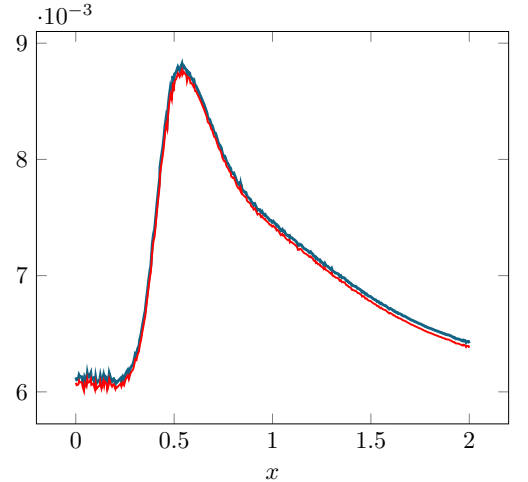

(a) Standard deviation of $u^{x}$

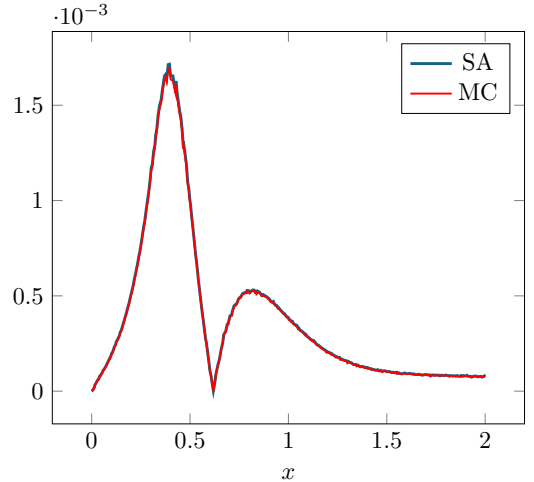

(b) Standard deviation of $u^{y}$

Figure 6: Standard deviation on the horizontal cross section $y=0.2$. Comparison between Monte Carlo (MC) and the SA estimate.

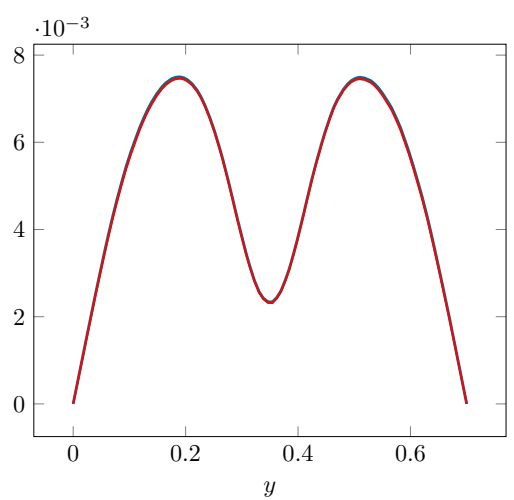

(a) Standard deviation of $u^{x}$

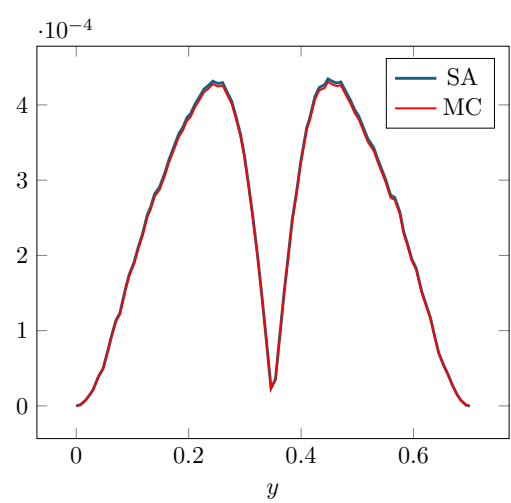

(b) Standard deviation of $u^{y}$

Figure 7: Standard deviation on the vertical cross section $x=1$. Comparison between Monte Carlo and the SA estimate.

of the state were necessary. In Figures 6-7, we compare the variance estimated using the sensitivities and the one computed with Monte Carlo on two cross sections of the domain. As one can see, the two strategies give very similar results. In Figures 8-9, we show the confidence intervals computed according to (20) for $\alpha=0.05$ : in blue the confidence intervals are obtained with the average and variance estimated with SA, in red with Monte Carlo.

For this test case, the first order approximations provided by the SA are more than satisfactory: with only two simulations, we obtain results comparable to the ones obtained with the Monte Carlo approach, which requires 1300 simulations.

\subsection{Unsteady test case}

We now consider a case with a higher Reynolds number $(R e=100$, which corresponds to $\left.\mu_{A}=1\right)$; thus, we are in the range in which a Von Karman vortex street occurs. In this situation, it is reasonable to assume that the velocity behaves as follows:

$$
\mathbf{u}(\mathbf{x}, t ; a) \simeq \sum_{k=0}^{N} \mathbf{u}_{0, k}(\mathbf{x} ; a) \cos \left(\omega_{k}(a) t\right)
$$




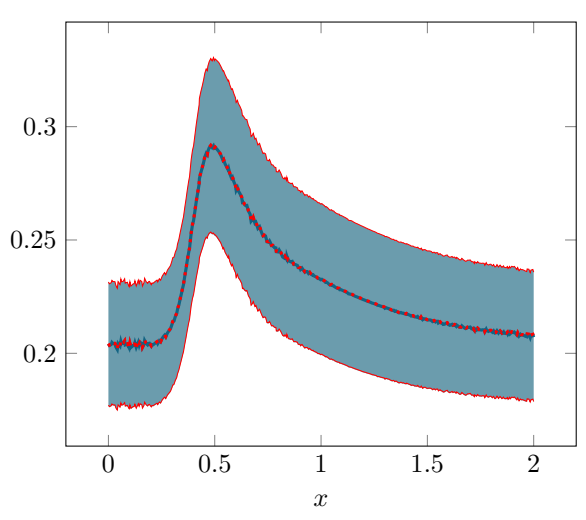

(a) Confidence interval for $u^{x}$

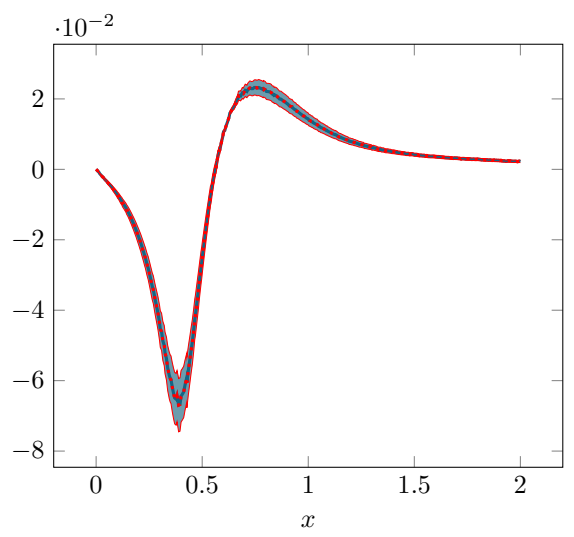

(b) Confidence interval for $u^{y}$

Figure 8: Confidence intervals on the horizontal cross section $y=0.2$, with $\alpha=0.05$. Comparison between Monte Carlo and the SA approaches.

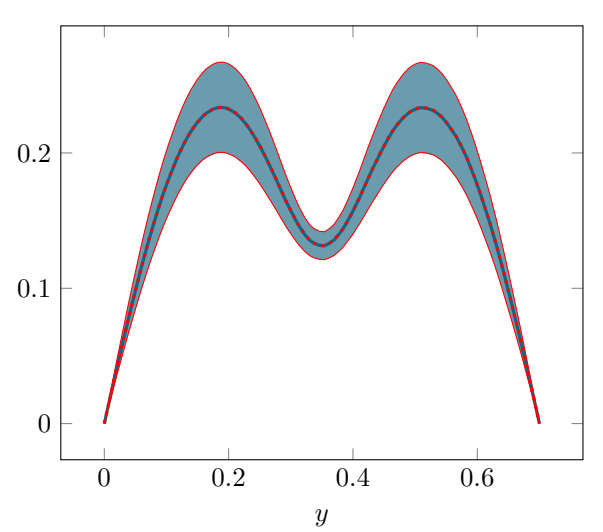

(a) Confidence interval for $u^{x}$

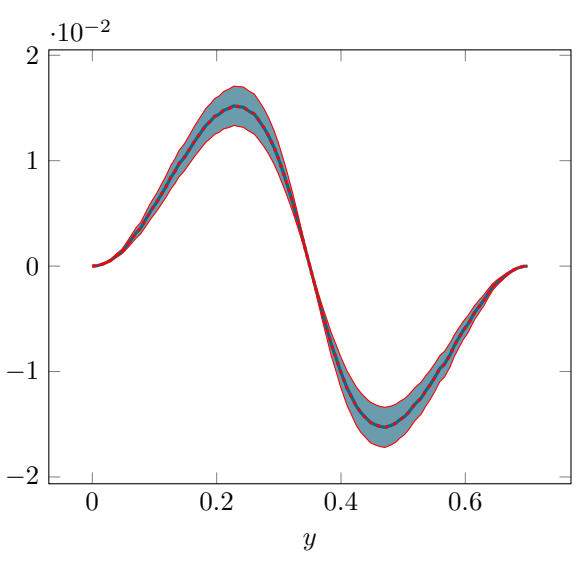

(b) Confidence interval for $u^{y}$

Figure 9: Confidence intervals on the vertical cross section $x=1$, with $\alpha=0.05$. Comparison between Monte Carlo and the SA approaches. 

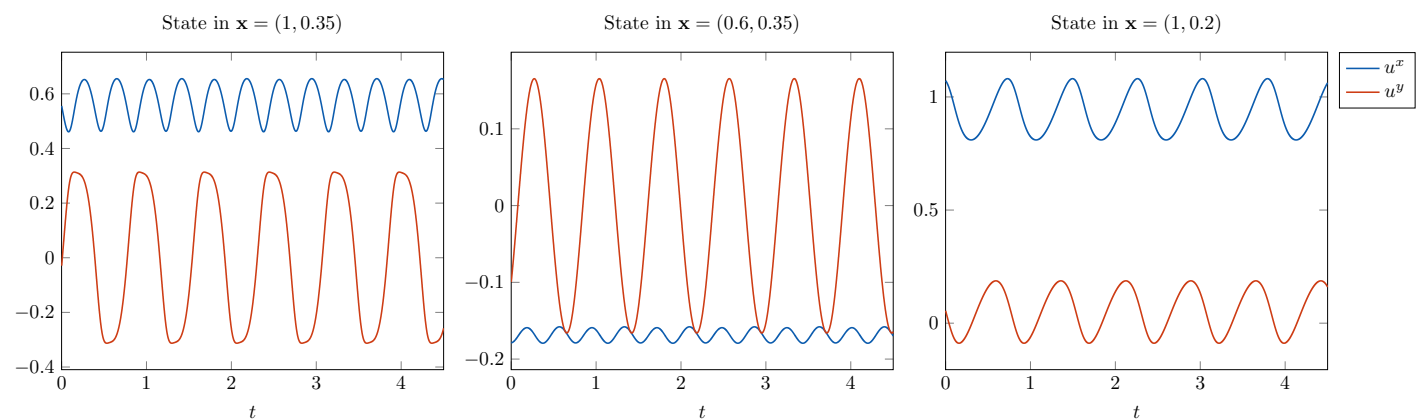

Figure 10: Velocity field in three different points of the domain with respect to time.
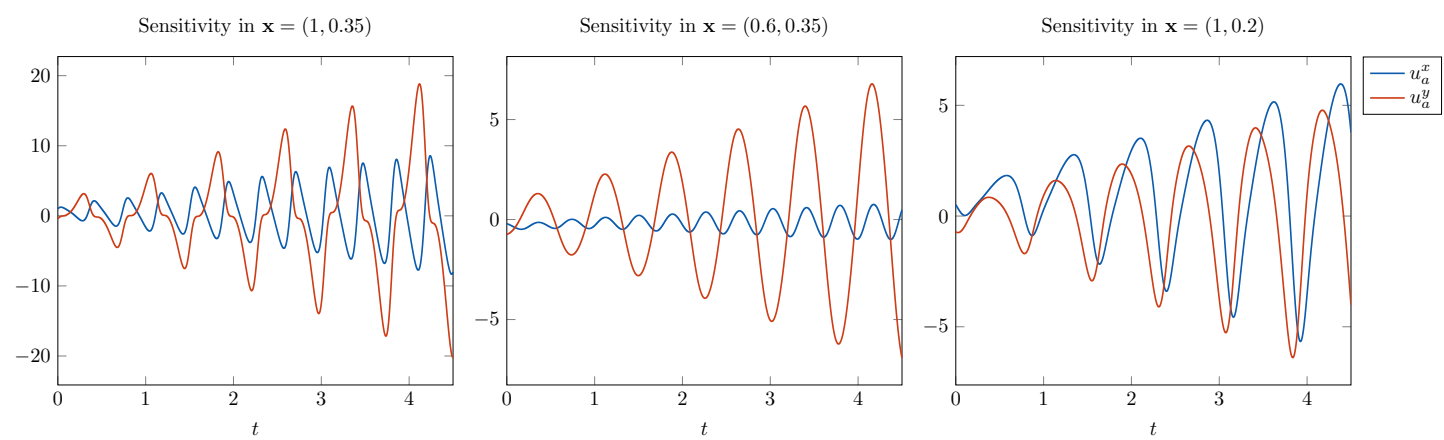

Figure 11: Sensitivity of the velocity field in three different points of the domain with respect to time.

We remark that (22) does not solve (1) exactly: some terms have been neglected. Although, from physical knowledge of the phenomenon and looking at numerical results downstream to the obstacle, we can say that the hypothesis (22) is reasonable and that $N$ can be as small as 2 or 3 .

To simulate this test case, we use the following procedure: first, we simulate the state until it reaches a periodic solution of the form (22); then, this state is injected into the sensitivity equations and the sensitivity simulation is run.

We remark that by differentiating (22) with respect to the parameter $a$, one obtains the following behaviour for the sensitivity:

$$
\mathbf{u}_{a}(\mathbf{x}, t ; a)=\sum_{k=0}^{N} \mathbf{u}_{0, a, k}(\mathbf{x} ; a) \cos \left(\omega_{k}(a) t\right)-t \sum_{k=0}^{N} \mathbf{u}_{0, k}(\mathbf{x} ; a) \omega_{k}^{\prime}(a) \sin \left(\omega_{k}(a) t\right)
$$

We can distinguish two contributions: the first sum corresponds to the influence that the input parameter $a$ has on the amplitude of the oscillations $\mathbf{u}_{0, k}$, the second sum corresponds to the influence on the the frequency $\omega(a)$. The second effect leads to a sensitivity that grows linearly in time and difficult to work with. This problem is described in [HEPB04] for a similar test case: to deal with it, they chose a pulsating inflow velocity, which imposes the frequency of vortex shedding (i.e. $\omega_{k}$ does not depend on $a$ ). However, this choice is not suitable for all applications. This is why, in the next subsection, we propose a filter to recover the first part of the sensitivity (23).

In Figures 10-11, we show the state and sensitivity in different points of the domain: as one can see, hypotheses (22) and (23) are verified. 

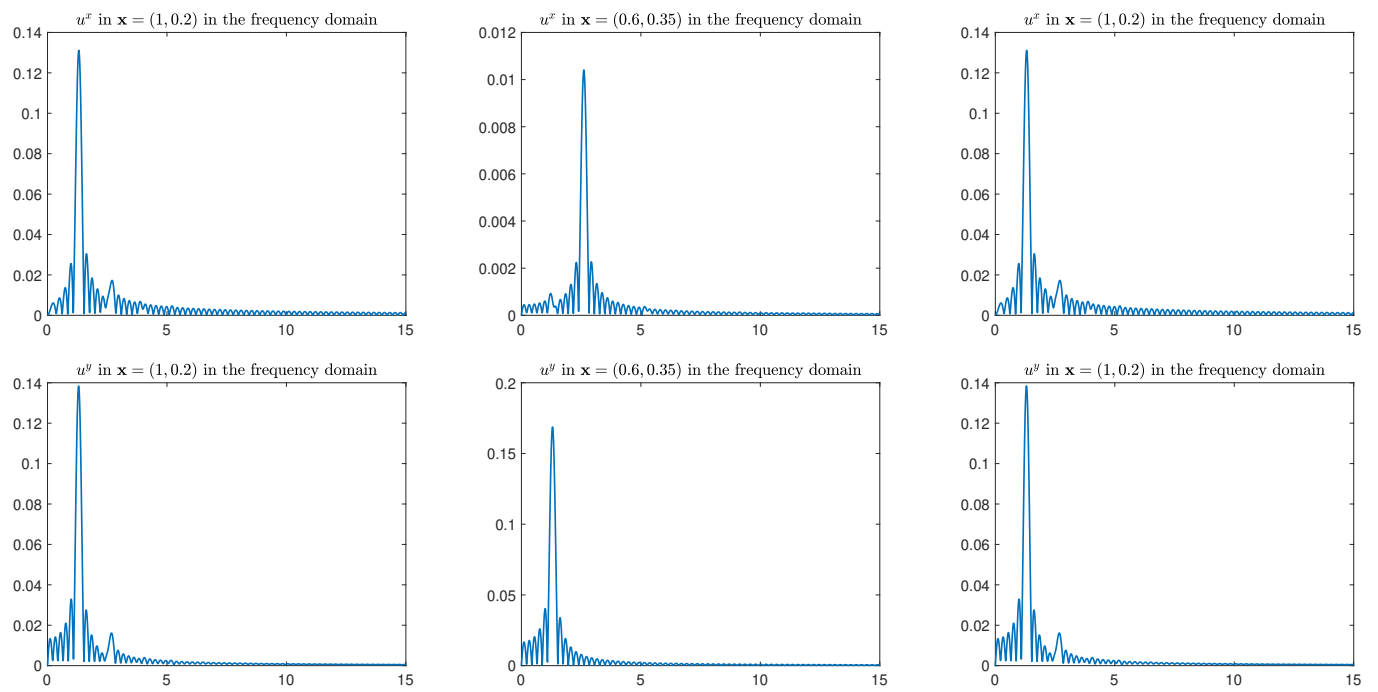

Figure 12: Fourier transform of the velocity in three different points. Top row: horizontal component. Bottom row: vertical component.

\subsubsection{Filter}

In this subsection, we propose a filter to recover the bounded part of the sensitivity

$$
\mathbf{h}(\mathbf{x}, t ; a):=\sum_{k=0}^{N} \mathbf{u}_{0, a, k}(\mathbf{x} ; a) \cos \left(\omega_{k}(a) t\right) .
$$

To the best of our knowledge, this is the first work in which a filtering procedure is proposed in this context. We focus on this part of the sensitivity because, for industrial applications, the variance of the amplitude of the oscillations is more interesting than the variance of their phase.

We suppose that the ratio between the $\omega_{k}$ is rational, i.e. that the state (22) and $\mathbf{h}$ are periodic in time. By computing the discrete Fourier transform of the state we can see that not only this is true, but also that we have a fundamental frequency $\bar{\omega}$ and then multiples of that, $2 \bar{\omega}, 3 \bar{\omega}$. In Figure 12, only the second harmonic $2 \bar{\omega}$ is visible. One can identify the period $T$ of the state using the Fourier transform, and therefore $\forall t$ the following equality holds:

$$
\int_{t-T}^{t} \frac{\mathbf{u}_{a}(\mathbf{x}, s ; a)}{s} d s=\int_{t-T}^{t} \frac{\mathbf{h}(\mathbf{x}, s ; a)}{s} d s=: \mathcal{I}(t) .
$$

The left-hand side of (24) can be computed numerically. To obtain $\mathbf{h}(\mathbf{x}, t ; a)$, one can compute the derivative of $\mathcal{I}(t)$ and use the fact that $\mathbf{h}$ is $T$-periodic in time:

$$
\frac{d \mathcal{I}}{d t}=\frac{\mathbf{h}(\mathbf{x}, t ; a)}{t}-\frac{\mathbf{h}(\mathbf{x}, t ; a)}{T-t}
$$

and finally one has

$$
\mathbf{h}(\mathbf{x}, t ; a)=\frac{t(T-t)}{T} \frac{d \mathcal{I}}{d t} .
$$

The derivative (25) can be computed numerically by finite differences. In Figure 13, we applied the filter just described to a case where $\mathbf{u}_{a}$ and $\mathbf{h}$ are known analytically: as one can see in the left Figure, the filter precision degrades with time. However, since we know that $\mathbf{h}$ is periodic, one can consider the first period and repeat it, which is what is shown in the right Figure. Of course, this leads to a small jump where the two periods are glued together. 

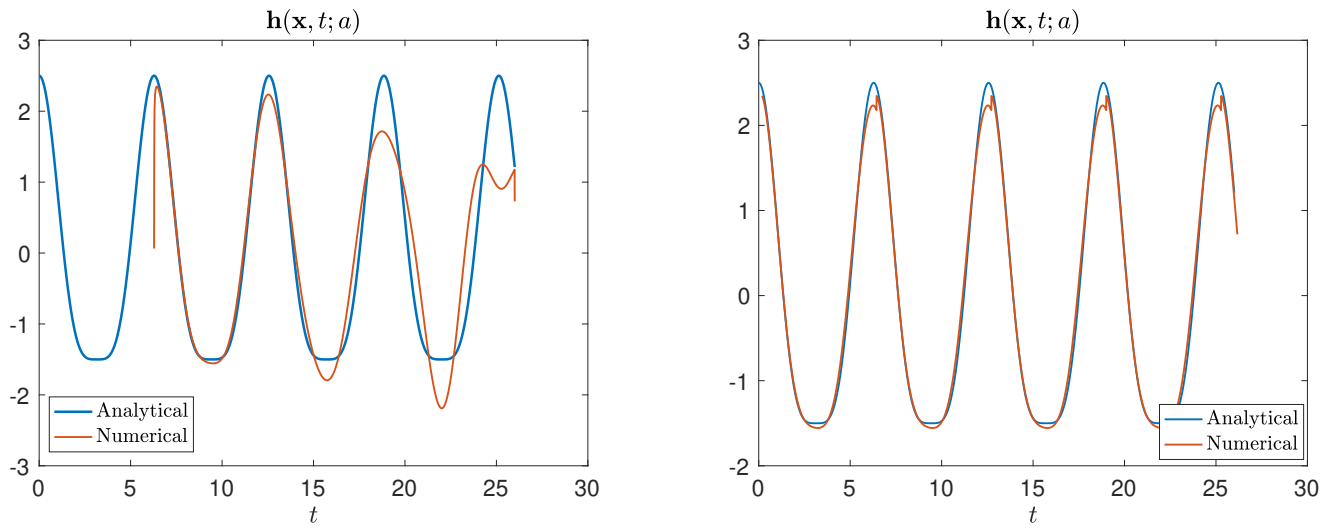

Figure 13: Filter applied to an analytical case.

We remark that this filter could be build on multiple periods as well. However, some considerations on the computational costs are necessary: in order to filter on $N$ periods, one has to run the simulation for at least $N+1$ periods. We tested the filter on multiple periods and the differences observed do not justify the additional computational cost for this test case. The following results are obtained using a one-period filter.

In Figure 14, we show the results of the filter applied to the sensitivities shown previously (Figures 10-11). The sensitivities obtained in this way can now be used for different applications such as, for instance, the computation of a confidence interval as done in the previous section (cf. Figure 15). However, one must be careful, as the filtered sensitivities take into account only the variance of the amplitude of the oscillations and not the variance of the frequency.

\section{Conclusion}

In this paper, we propose an efficient computational strategy to deal with problems of uncertainty propagation for the Navier-Stokes equations. First, the state equations are presented, and the sensitivity equations are derived according to the continuous sensitivity equation method. Stability estimates in $L^{\infty}\left(0, T ; L^{2}(\Omega)\right) \cap L^{2}\left(0, T ; H^{1}(\Omega)\right)$ for both the state and the sensitivity are rigorously proven. We homogenise the Dirichlet inflow boundary conditions using an auxiliary function which is explicitly computed. A FEV numerical scheme is designed for the sensitivity and implemented in the open-source code TrioCFD. A classical test case of flow past a square-section cylinder is investigated in two different regimes: a steady case $(R e=25)$ and a Karman vortex street $(R e=100)$. For both cases, the sensitivity is used to estimate the variance of the velocity field, and $95 \%$ confidence intervals are computed. In the steady case, a detailed comparison with a Monte Carlo method is performed: the results of the sensitivity based method are extremely accurate, and the computational gain is significant. The unsteady test case raises an issue: due to the periodic nature of the velocity, the sensitivity grows in time and is therefore unusable. We propose a new procedure, which consists in filtering the different contributions to the sensitivity in order to recover its physical parts. The filtered sensitivity is then used to compute the confidence intervals.

Questions such as the investigation of different parameters of interest, 3-dimensional computations, the addition of the energy equation to deal with the temperature and the extension to the unsteady Reynolds-Averaged Navier-Stokes (URANS) equations for the study of turbulent flows will be addressed in future works, to tackle more realistic problems. 
$u_{a}^{x}$ in $\mathbf{x}=(1,0.35)$

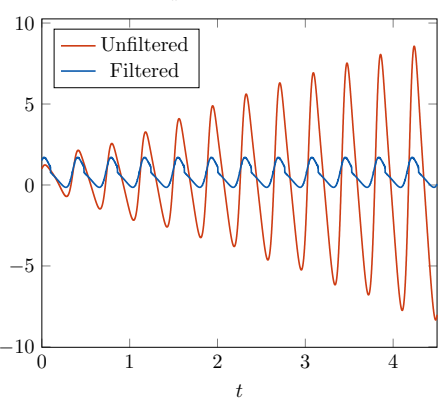

$u_{a}^{y}$ in $\mathbf{x}=(1,0.35)$

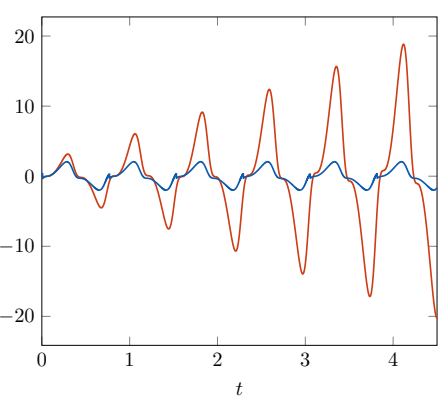

$u_{a}^{x}$ in $\mathbf{x}=(0.6,0.35)$

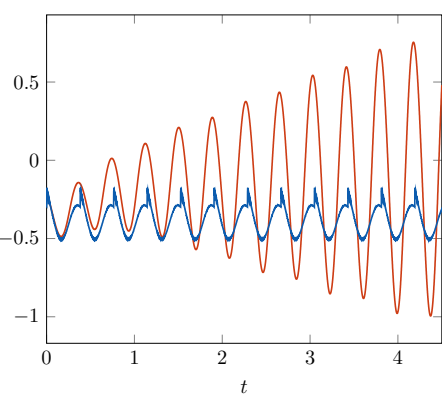

$u_{a}^{y}$ in $\mathbf{x}=(0.6,0.35)$

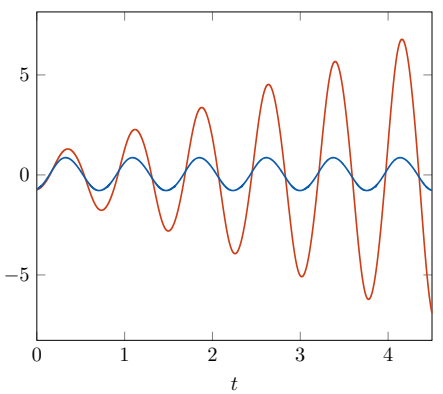

$u_{a}^{x}$ in $\mathbf{x}=(1,0.2)$

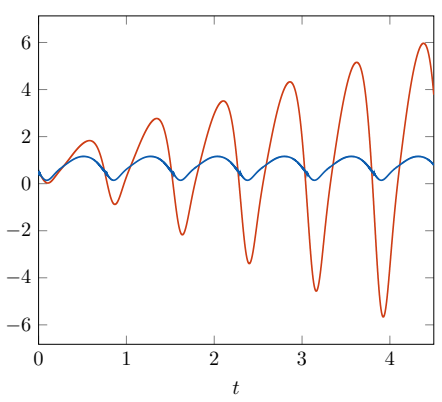

$u_{a}^{y}$ in $\mathbf{x}=(1,0.2)$

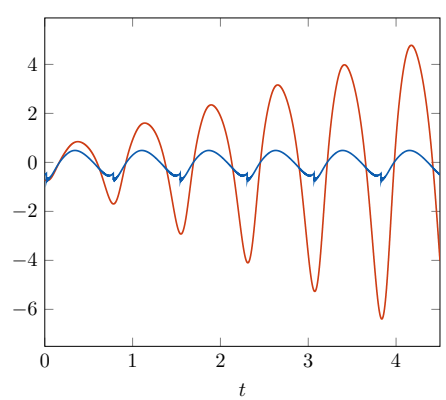

Figure 14: Filter applied to the sensitivity of the velocity in three different points of the domain. Top row: horizontal component. Bottom row: vertical component.
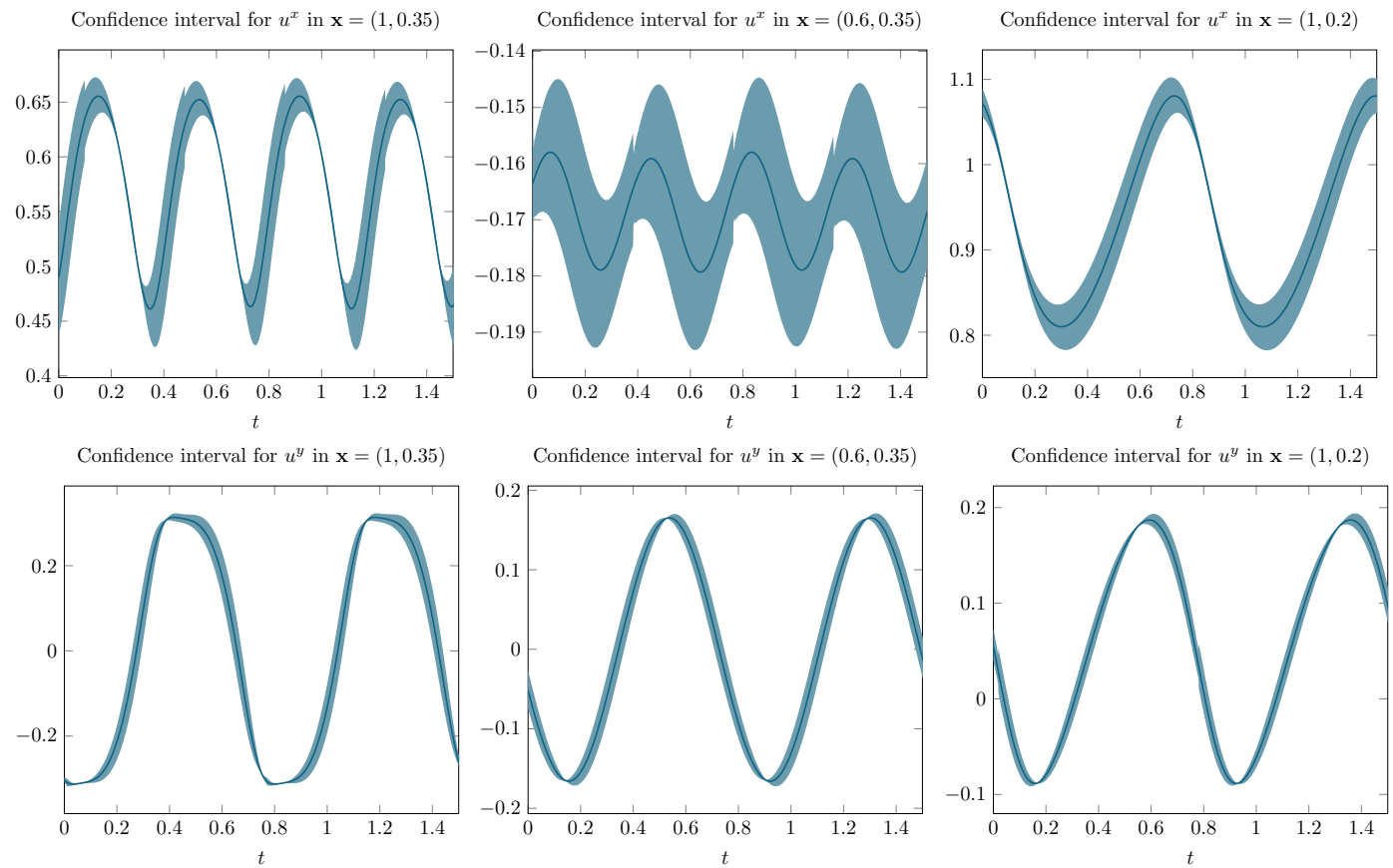

Figure 15: Confidence intervals $(\alpha=0.05)$ for the velocity in three different points of the domain. Top row: horizontal component. Bottom row: vertical component. 


\section{Appendix}

\section{A Computation of the auxiliary function $\mathbf{R}_{g}(x, y)$}

We need a vector field $\mathbf{R}_{g}(x, y)=\left(R_{g}^{x}(x, y), R_{g}^{y}(x, y)\right)^{t}$ which is solenoidal, smooth enough (at least continuous with $\nabla \mathbf{R}_{g} \in L^{\infty}(\Omega)$ ) and equal to $\mathbf{u}$ on the Dirichlet boundary, i.e. on $\Gamma_{i n} \cup \Gamma_{w}$. The same can be done for $\mathbf{u}_{a}$, in particular, for the inflow boundary condition used in this work (21).

First of all, one needs to define a function $g_{F}(y)$ with the following properties:

$$
\begin{gathered}
g_{F}(0)=g_{F}(\ell)=0, \\
g_{F}(y)=0 \forall y \in\left[\frac{\ell-d}{2}, \frac{\ell+d}{2}\right], \\
g_{F} \in C^{0}[0, \ell], \quad \partial_{y} g_{F} \in L^{\infty}[0, \ell] \\
\int_{0}^{\ell} g_{F}(y) d y=\int_{0}^{\ell} g(y) d y .
\end{gathered}
$$

This is easy and a function with these properties will be specified later. Let $\mathcal{H}(x)$ be a $C^{2}$ function such that

$$
\mathcal{H}(0)=1, \quad \mathcal{H}(x)=0 \forall x \in\left[x_{D}, L\right], \quad \mathcal{H}^{\prime}(0)=\mathcal{H}^{\prime}\left(x_{D}\right)=0 .
$$

Many different options are possible for $\mathcal{H}(x)$. Let $G(y)$ (respectively $G_{F}(y)$ ) be a primitive of $g(y)$ (respectively $\left.g_{F}(y)\right)$ :

$$
G(y)=\int_{0}^{y} g(s) d s \quad G_{F}(y)=\int_{0}^{y} g_{F}(s) d s .
$$

Proposition 3. The following vector field is solenoidal, continuous and equals $\mathbf{u}$ at the boundary:

$$
R_{g}^{x}(x, y)=g(y) \mathcal{H}(x)+g_{F}(y)(1-\mathcal{H}(x)) \quad R_{g}^{y}(x, y)=-\mathcal{H}^{\prime}(x)\left(G(y)-G_{F}(y)\right) .
$$

Furthermore, $\nabla \mathbf{R}_{g} \in L^{\infty}(\Omega)$ and $\left.\nabla \mathbf{R}_{g} \mathbf{n}\right|_{\Gamma_{\text {out }}}=0$.

Proof. (i) The field is solenoidal:

$$
\nabla \cdot \mathbf{R}_{g}=\partial_{x} R_{g}^{x}+\partial_{y} R_{g}^{y}=g(y) \mathcal{H}^{\prime}(x)-g_{F} \mathcal{H}^{\prime}(x)-\mathcal{H}^{\prime}(x)\left(g(y)-g_{F}(y)\right)=0 .
$$

(ii) The field is continuous because each component is a product and sum of continuous functions.

(iii) The field equals $\mathbf{u}$ on $\Gamma_{D}$.

(a) The $x$ component of $\mathbf{u}$ is equal to $g(y)$ on $\Gamma_{i n}$ and zero on $\Gamma_{D} \backslash \Gamma_{i n}$. On $\Gamma_{i n}$, we have:

$$
R_{g}^{x}(0, y)=g(y) \mathcal{H}(0)+g_{F}(y)(1-\mathcal{H}(0))=g(y),
$$

because $\mathcal{H}(0)=1$. On $\Gamma_{\text {top }}$ and $\Gamma_{\text {bottom }}$,

$$
R_{g}^{x}(\ell, y)=R_{g}^{x}(0, y)=0
$$

because $g(0)=g(\ell)=0$ and $g_{F}(0)=g_{F}(\ell)=0$. Finally, on the obstacle we have

$$
R_{g}^{x}(x, y)=g_{F}(y)
$$

which is zero $\forall y \in\left[\frac{\ell-d}{2}, \frac{\ell+d}{2}\right]$ thanks to property (26). 
(b) The $y$ component of $\mathbf{u}$ is zero on $\Gamma_{D}$. On $\Gamma_{i n}$ we have

$$
R_{g}^{y}(0, y)=0
$$

because $\mathcal{H}^{\prime}(0)=0$. On the bottom wall $y=0$, we have

$$
R_{g}^{y}(x, 0)=-\mathcal{H}^{\prime}(x)\left(G(0)-G_{F}(0)\right)=0,
$$

because $G(0)=G_{F}(0)=0$, from (28). On the top wall $y=\ell$ :

$$
R_{g}^{y}(x, \ell)=0,
$$

because $G(\ell)=G_{F}(\ell)$ thanks to the property (27). Finally, on the obstacle we have

$$
R_{g}^{y}(x, y)=0,
$$

because $\mathcal{H}^{\prime}(x)=0 \forall x \in\left[x_{D}, L\right]$.

(iv) $\nabla \mathbf{R}_{g} \in L^{\infty}(\Omega)$ and $\left.\nabla \mathbf{R}_{g} \mathbf{n}\right|_{\Gamma_{\text {out }}}=0$. The gradient of $\mathbf{R}_{g}$ is the following:

$$
\nabla \mathbf{R}_{g}(x, y)=\left[\begin{array}{cc}
\mathcal{H}^{\prime}(x)\left(g(y)-g_{F}(y)\right) & \left(g^{\prime}(y)-g_{F}^{\prime}(y)\right) \mathcal{H}(x)+g_{F}^{\prime}(y) \\
-\mathcal{H}^{\prime \prime}(x)\left(G(y)-G_{F}(y)\right) & -\mathcal{H}^{\prime}(x)\left(g(y)-g_{F}(y)\right)
\end{array}\right]
$$

which is in $L^{\infty}(\Omega)$ thanks to the hypotheses of regularity of $\mathcal{H}$ and $g_{F}$. Finally, on the outflow boundary the normal is $\mathbf{n}=(1,0)^{t}$. If we evaluate $(29)$ on $\Gamma_{\text {out }}$, i.e. $x=L$, we have:

$$
\nabla \mathbf{R}_{g}(L, y)=\left[\begin{array}{cc}
0 & g_{F}^{\prime}(y) \\
0 & 0
\end{array}\right]
$$

and therefore $\left.\nabla \mathbf{R}_{g} \mathbf{n}\right|_{\Gamma_{\text {out }}}=0$.

\section{Example of an auxiliary function for the state}

Let $g$ and its primitive $G$ be the following functions:

$$
g(y)=y(\ell-y), \quad G(y)=\frac{\ell}{2} y^{2}-\frac{1}{3} y^{3} .
$$

Then, we can define the function $g_{F}$ as follows:

$$
g_{F}(y)= \begin{cases}k y\left(\frac{\ell-d}{2}-y\right) & y \in\left[0, \frac{\ell-d}{2}\right), \\ 0 & y \in\left[\frac{\ell-d}{2}, \frac{\ell+d}{2}\right], \\ k(\ell-y)\left(y-\frac{\ell+d}{2}\right) & y \in\left(\frac{\ell+d}{2}, \ell\right]\end{cases}
$$

where $k$ is chosen in order to respect the property (27) and it is $k=\frac{4 \ell^{3}}{(\ell-d)^{3}}$. The primitive $G_{F}$ is:

$$
G_{F}(y)= \begin{cases}k \frac{\ell-d}{4} y^{2}-k \frac{y^{3}}{3} & y \in\left[0, \frac{\ell-d}{2}\right), \\ \frac{\ell}{12} & y \in\left[\frac{\ell-d}{2}, \frac{\ell+d}{2}\right], \\ \frac{\ell}{12}-k\left(\frac{y^{3}}{3}-\frac{3 \ell+d}{4} y^{2}+\frac{\ell(\ell+d)}{2} y\right)-k\left(\frac{\ell+d}{2}\right)^{2}\left(\frac{d-5 \ell}{12}\right) & y \in\left(\frac{\ell+d}{2}, \ell\right] .\end{cases}
$$

Finally, we chose the following function for $\mathcal{H}(x)$ :

$$
\mathcal{H}(x)= \begin{cases}2\left(\frac{x}{x_{D}}\right)^{3}-3\left(\frac{x}{x_{D}}\right)^{2}+1 & x \in\left[0, x_{D}\right], \\ 0 & x \in\left(x_{D}, L\right],\end{cases}
$$

which has all the required properties. The auxiliary function just computed is shown in Figure 16. Figure 17 shows the two components of $\mathbf{R}_{g}, R_{g}^{x}$ and $R_{g}^{y}$. 


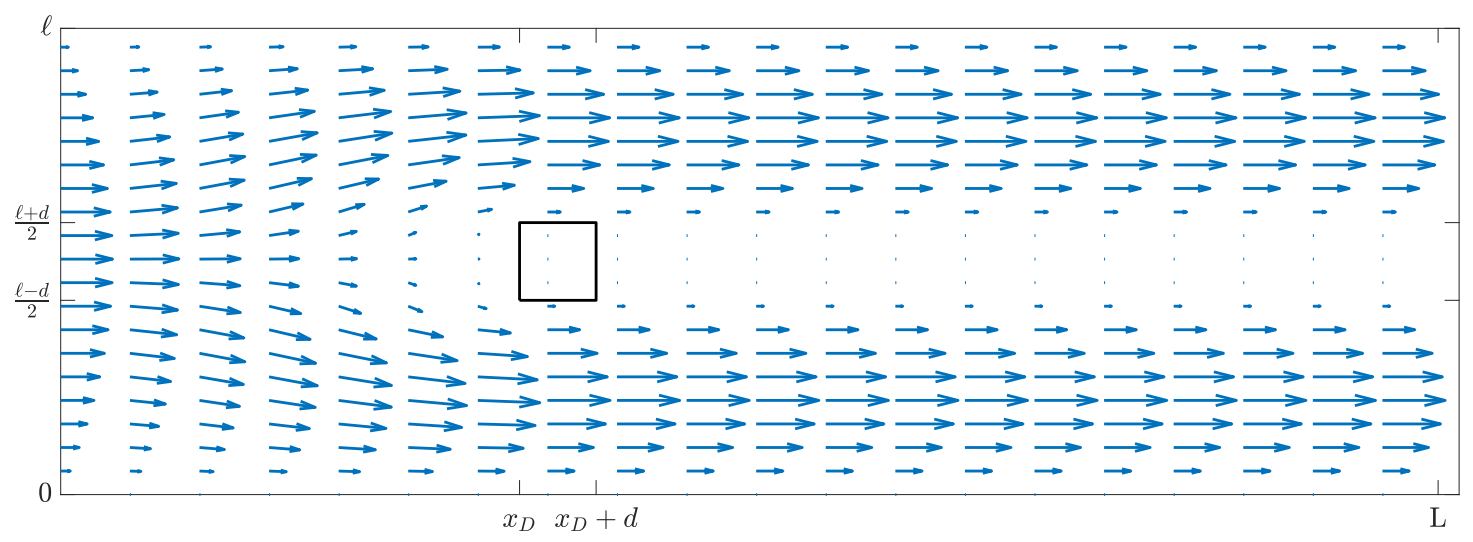

Figure 16: Solenoidal field from the example.

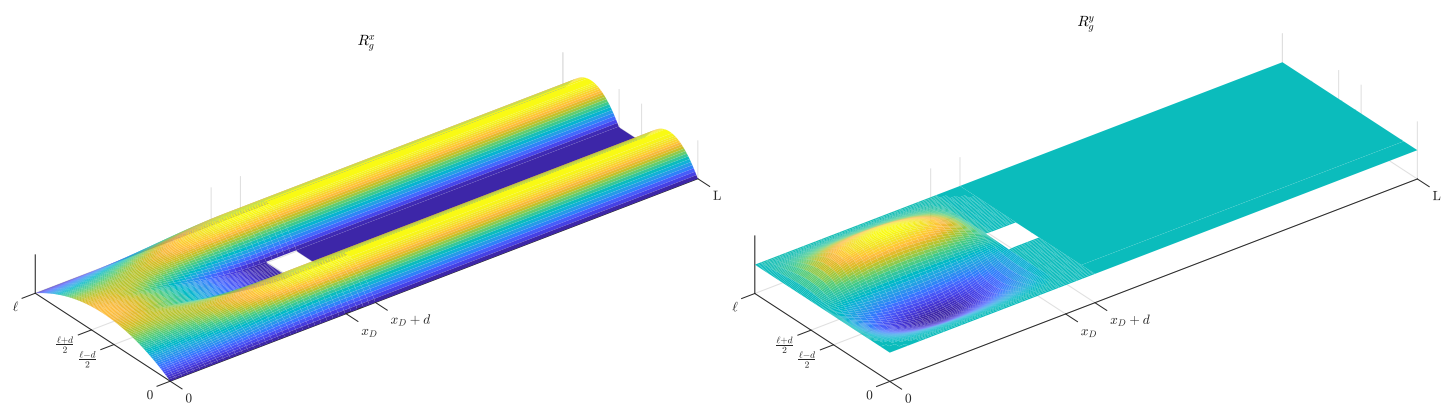

Figure 17: Solenoidal field from the example, $x$ and $y$ components. 


\section{References}

[ABF15] P.-E. Angeli, U. Bieder, and G. Fauchet. Overview of the TrioCFD code: main features, $\mathrm{V} \& \mathrm{~V}$ procedures and typical applications to nuclear engineering. Nureth. 16. Chicago, USA, page 252, 2015.

[AC13] Remi Abgrall and Pietro Marco Congedo. A semi-intrusive deterministic approach to uncertainty quantification in non-linear fluid flow problems. Journal of Computational Physics, 235:828-845, 2013.

[APFC17] P. E. Angeli, M. A. Puscas, G. Fauchet, and A. Cartalade. FVCA8 Benchmark for the Stokes and Navier-Stokes equations with the TrioCFD code - Benchmark session. In International Conference on Finite Volumes for Complex Applications, pages 181-202. Springer, 2017.

[BB97] J. Borggaard and J. Burns. A PDE sensitivity equation method for optimal aerodynamic design. Journal of Computational Physics, 136(2):366 - 384, 1997.

[Bre10] H. Brezis. Functional analysis, Sobolev spaces and partial differential equations. Springer Science \& Business Media, 2010.

[BZD00] J. Breuer, M and Bernsdorf, T. Zeiser, and F. Durst. Accurate computations of the laminar flow past a square cylinder based on two different methods: lattice-Boltzmann and finite-volume. International journal of heat and fluid flow, 21(2):186-196, 2000.

[CDF18] C. Chalons, R. Duvigneau, and C. Fiorini. Sensitivity analysis and numerical diffusion effects for hyperbolic PDE systems with discontinuous solutions. The case of barotropic Euler equations in Lagrangian coordinates. SIAM Journal on Scientific Computing, 40(6):A3955-A3981, 2018.

[CFL67] R. Courant, K. Friedrichs, and H. Lewy. On the partial difference equations of mathematical physics. IBM journal of Research and Development, 11(2):215234, 1967.

[Cho68] A. J. Chorin. Numerical solution of the navier-stokes equations. Mathematics of computation, 22(104):745-762, 1968.

[DC] DEN-CEA. TRUST TrioCFD code.

[Del14] C. Delenne. Propagation de la sensibilité dans les modèles hydrodynamiques., 2014.

[DP05] R. Duvigneau and D. Pelletier. Evaluation of nearby flows by a shape sensitivity equation method. In 43rd AIAA Aerospace Sciences Meeting and Exhibit, page 127,2005 .

[DP06] R. Duvigneau and D. Pelletier. A sensitivity equation method for fast evaluation of nearby flows and uncertainty analysis for shape parameters. International Journal of Computational Fluid Dynamics, 20(7):497-512, 2006.

[DPB06] R. Duvigneau, D. Pelletier, and J. Borggaard. An improved continuous sensitivity equation method for optimal shape design in mixed convection. Numerical Heat Transfer, Part B: Fundamentals, 50(1):1-24, 2006.

[DPL13] Bruno Després, Gaël Poëtte, and Didier Lucor. Robust uncertainty propagation in systems of conservation laws with the entropy closure method. In Uncertainty quantification in computational fluid dynamics, pages 105-149. Springer, 2013.

[Emo92] P. Emonot. Méthodes de volumes éléments finis: applications aux équations de Navier Stokes et résultats de convergence. PhD thesis, Lyon 1, 1992.

[FCD19] C. Fiorini, C. Chalons, and R. Duvigneau. A modified ensitivity equation method for Euler equations in presence of shocks. Numerical methods for partial differential equations, 2019.

[Fio18] C. Fiorini. Sensitivity analysis for nonlinear hyperbolic systems of conservation laws. PhD thesis, Université Paris Saclay, 2018. 
[Gun03] M. D. Gunzburger. Perspectives in flow control and optimization, volume 5. Siam, 2003.

[Hei03] S. Heib. Nouvelles discrétisations non structurées pour des écoulements de fluides à incompressibilité renforcée. PhD thesis, Paris 6, 2003.

[HEPB04] H. Hristova, S. Etienne, D. Pelletier, and J. Borggaard. A continuous sensitivity equation method for time-dependent incompressible laminar flows. International Journal for Numerical Methods in Fluids, 50:817-844, 2004.

[JP12] J. Jacod and P. Protter. Probability essentials. Springer Science \& Business Media, 2012.

[KM06] O.M. Knio and O.P. Le Maitre. Uncertainty propagation in CFD using polynomial chaos decomposition. Fluid Dynamics Research, 38(9):616-640, September 2006.

[QSS10] A. Quarteroni, R. Sacco, and F. Saleri. Numerical mathematics, volume 37. Springer Science \& Business Media, 2010.

[Ray07] J-P Raymond. Stokes and Navier-Stokes equations with nonhomogeneous boundary conditions. Annales de l'Institut Henri Poincaré - Analyse Non Linéaire, 24(6):921-951, 2007.

[RC13] C. Robert and G. Casella. Monte Carlo statistical methods. Springer Science \& Business Media, 2013.

[Tem68] R. Temam. Une méthode d'approximation de la solution des équations de navierstokes. Bulletin de la Société Mathématique de France, 96:115-152, 1968.

[Vol14] J. Volker. Lecture notes on numerical methods for incompressible flow problems II, Chapter 4, 2014.

[Wal03] R. Walters. Towards stochastic fluid mechanics via polynomial chaos. In $41 s t$ AIAA Aerospace Sciences Meeting and Exhibit, Reno, USA, 2003.

[WH02] R. W. Walters and L. Huyse. Uncertainty analysis for fluid mechanics with applications. Technical report, National aeronautics and space administration, Hampton, VA, Langley research center, 2002.

[XK03] D. Xiu and George E. Karniadakis. Modeling uncertainty in flow simulations via generalized polynomial chaos. Journal of computational physics, 187(1):137-167, 2003. 